\title{
Data on Particles and Resonant States*
}

\author{
ARTHUR H. ROSENFELD, ANGELA BARBARO-GALTIERI, WALTER H. BARKAS, $†$ PIERRE L. BASTIEN, \\ JANOS KIRZ \\ Lawrence Radiation Laboratory, University of California, Berkeley, California \\ MATTS ROOS \\ CERN, Geneva, Switzerland and Institute for Theoretical Physics, University of Copenhagen, Copenhagen, Denmark
}

\begin{abstract}
Data and references on properties of particles and resonances are compiled, tested for consistency, and summarized in tables and wallet cards. This is an updating of the Reviews of Modern Physics article of October 1964, and some new quantities have been included in the tables.
\end{abstract}

This data survey is an updating of that of one year ago. ${ }^{1}$ To save space we discuss only the changes since then, and omit the descriptions of the tables and compiling procedures. However, we do want to re-emphasize the warning carried by the entries " $\times$ Scale $=.$. ." As an example, consider the $A 2$ meson, whose mass is listed as $1324 \pm 9 \times$ Scale $=2.5 \mathrm{MeV}$. This means that the masses used in arriving at the weighted average of $1324 \pm 9 \mathrm{MeV}$ are inconsistent, and have a chi-squared larger than expected by a factor of $2.5^{2}$. In UCRL $8030^{2}$ we present ideograms of all the sets of input data which have abnormally large chi squareds, and the ideogram for the $A 2$ mass is double-humped, showing that the $9-\mathrm{MeV}$ calculated error of the average is absurdly unrealistic. The reader can look at the ideogram and decide for himself which experiments to bet on. If he chooses not to do this, he should at least take the point of view that all the input errors are equally underestimated (by a factor of 2.5), and take the calculated error to be $9 \mathrm{MeV} \times 2.5$, i.e., about $22 \mathrm{MeV}$.

Wallet cards in two sizes are available from Lawrence Radiation Laboratory, University of California, Berkeley, California.

\section{CHANGES IN TABLE S (STABLE PARTICLES)}

We have added magnetic moments to Table $\mathrm{S}$, and decay parameters of hyperons to Table S-Decay.

A new measurement by Shafer, Crowe, and Jenkins (SHAFER 65) has reduced the error on the charged pion mass from 50 to $15 \mathrm{keV}$. This result affects the mass of all the other mesons and hyperons to a small extent. For an up-to-date review, see the article by Barkas. ${ }^{3}$

\footnotetext{
* This work is done under the auspices of the U.S. Atomic Energy Commission.

$\dagger$ Present address: University of California at Riverside, California.

‡Present address: Massachusetts Institute of Technology, Cambridge, Massachusetts.

1 A. H. Rosenfeld, A. Barbaro-Galtieri, W. H. Barkas, P. L. Bastien, J. Kirz, and Matts Roos, Rev. Mod. Phys. 36, 977 (1964).

${ }^{2}$ Rosenfeld et al., UCRL 8030 revised (unpublished). Available for $\$ 2.00$ from the Clearinghouse for Federal Scientific and Technical Information, National Bureau of Standards, U.S. Department of Commerce, Springfield, Virginia.

${ }^{3}$ W. H. Barkas, Ann. Rev. Nucl. Sci. 15 (1965, to be published).
}

\section{CHANGES IN THE IMESON TABLE}

In the meson table we have listed several states for which it has not been shown that they have welldefined quantum numbers $(D, E, A 1, B, \kappa, C)$. Alternative explanations have been put forward for the $A 1,,^{4-7}$ the $B,{ }^{8}$ and the $\kappa .^{9}$

Because of their current interest, we have added information on possible $C$-violating decay modes.

\section{CHANGES IN THE BARYON TABLE}

New useful quantities have been added in this table. In the second column of the table we give both kinetic energy and momentum of the $\pi$ or $K$ beam (incident on a proton), out of which these resonances can be directly formed. In addition to the mass squared of the resonance, we give now also $\Gamma\left(M^{2}\right)$. On a mass-squared scale, this quantity is equivalent to the full width of the resonance; i.e., $\Gamma\left(M^{2}\right)=2 M \Gamma(M)$.

\section{SU(3) ASSIGNMENTS}

A large fraction of the particles with known spin and parity has been successfully grouped into $\mathrm{SU}(3)$ multiplets.

Among the baryons the $N, \Lambda, \Sigma$, and $\Xi$ are assigned to a $J^{P}=\frac{1}{2}+$ octet, satisfying the Gell-Mann-Okubo mass formula ${ }^{10}$ :

$$
\frac{1}{2}\left(M_{N}+M_{\Xi}\right)=\frac{1}{4}\left(3 M_{\Lambda}+M_{\Sigma}\right) \text {. }
$$

The $N_{\frac{s}{2}}^{*}(1238), Y_{1}^{*}(1385), \Xi^{*}(1530)$, and $\Omega^{-}$are assigned to a $\frac{3}{2}+$ decuplet satisfying the equal mass spacing rule.

Among the mesons nine $0^{-}$states $\left(\pi, K, \eta, X^{0}\right)$, and

${ }^{4}$ R. T. Deck, Phys. Rev. Letters 13, 169 (1965).

${ }^{5}$ G. Goldhaber, Proc. Coral Gables Conference, 1965, p. 34

${ }^{6}$ U. Maor and T. A. O'Halloran, Jr., Phys. Letters 15, 281 (1965).

${ }^{7}$ N. P. Chang, Phys. Rev. Letters 14, 806 (1965).

${ }^{8}$ G. Goldhaber et al., Phys. Rev. Letters 15, 118 (1965).

${ }^{9}$ Melvin Month, University of Illinois preprint, 1965 (unpublished).

${ }^{10}$ M. Gell-Mann, California Institute of Technology Report, CTSL-20 (1961) ; S. Okubo, Progr. Theoret. Phys. (Kyoto) 27, 949 (1962). 
TABLES FROM UCRL-8030(rev.)

Table S - Stable particles

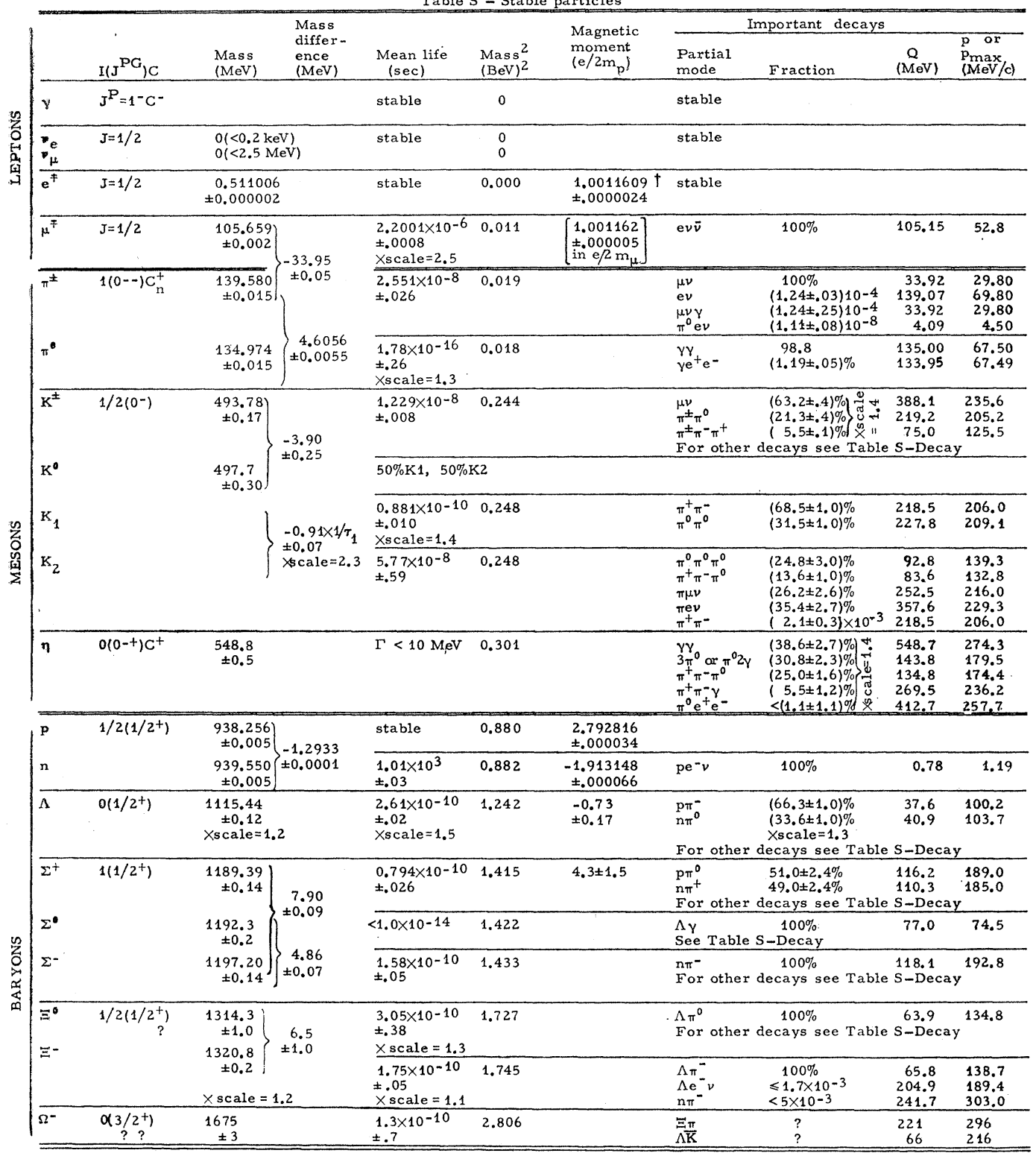

$t_{\text {In units of }}\left(e / 2 \mathrm{~m}_{e}\right)$.

A. H. Rosenfeld, A. Barbaro-Galtieri, W. H. Barkas, P. L. Bastien, J. Kirz, and M. Roos,

UCRI-8030 - Part I, August 1965. 
Table S-Decay

An Appendix to Table $S$ for decay parameters and branching fractions

\begin{tabular}{|c|c|c|c|c|c|c|c|c|}
\hline & $\begin{array}{c}\text { Partial } \\
\text { mode }\end{array}$ & Fraction & $\begin{array}{c}\mathrm{Q} \\
(\mathrm{MeV})\end{array}$ & $\begin{array}{c}\mathrm{p} \text { or } \mathrm{p}_{\max } \\
(\mathrm{MeV} / \mathrm{c})\end{array}$ & $a^{\dagger}$ & $\beta^{\dagger}$ & $\gamma^{\dagger}$ & $\Delta^{\dagger}$ \\
\hline$\overline{\mathrm{K}^{ \pm}}$ & 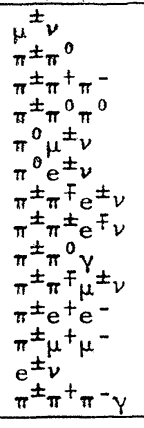 & $\begin{array}{c}(63.2 \pm .4) \% \\
(21.3 \pm .4) \% \\
(5.52 \pm .08) \% \\
(1.68 \pm .05) \% \\
(3.4 \pm .2) \% \\
(4.9 \pm .2) \% \\
(4.3 \pm .3) \\
<0.1 \times 10^{-5} \\
(2.2 \pm 0.7) 10 \\
\leqslant 1.2 \times 10^{-5} \\
<1.1 \times 10^{-6} \\
<3 \times 10^{-6} \\
<1.6 \times 10^{-3} \\
(9 \pm 4) 10^{-5} \\
<\end{array}$ & $\begin{array}{r}388.1 \\
219.2 \\
75.0 \\
84.3 \\
253.1 \\
358.3 \\
214.1 \\
214.1 \\
219.2 \\
109.0 \\
353.2 \\
142.9 \\
493.3 \\
75.0 \\
\end{array}$ & $\begin{array}{l}235.6 \\
205.2 \\
125.5 \\
133.0 \\
215.2 \\
228.4 \\
203.5 \\
203.5 \\
205.2 \\
151.1 \\
227.2 \\
171.9 \\
246.9 \\
125.5 \\
\end{array}$ & & & & 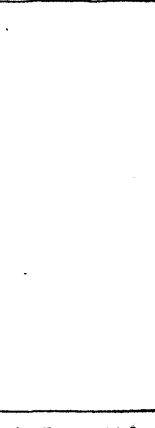 \\
\hline$\Lambda$ & $\begin{array}{l}\mathrm{p} \pi^{-} \\
n \pi^{\circ} \\
\mathrm{p} \mu \nu \\
\mathrm{pev}\end{array}$ & $\begin{array}{l}(66.3 \pm 1.0) \%) \times \text { scale } \\
(33.6 \pm 1.0) \%)=1.2 \\
(1.5 \pm 1.2) 10^{-4} \\
(0.88 \pm .08) 10^{-3} \\
\times \text { scale }=1.3\end{array}$ & $\begin{array}{r}37.6 \\
40.9 \\
71.5 \\
176.7\end{array}$ & $\begin{array}{l}100.2 \\
103.7 \\
130.8 \\
163.1\end{array}$ & $+0.659 \pm 0.047$ & : & & $(15 \pm 20)^{\circ}$ \\
\hline$\Sigma^{+}$ & $\begin{array}{l}\mathrm{pr}^{0} \\
\mathrm{~nm} \pi^{+} \\
\mathrm{n} \pi^{+} \gamma \\
\Lambda \mathrm{e}^{+} v \\
\mathrm{py} \\
\mathrm{nu}{ }^{+} v \\
n \mathrm{e}^{+} v\end{array}$ & $\begin{aligned} &(51.0 \pm 2.4) \% \\
&(49.0 \pm 2.4) \% \\
& \approx 0.2 \times 10^{-4} \\
& \approx 0.2 \times 10^{-4} \\
&(3.7 \pm 0.8) 10^{-4} \\
&<1.1 \times 10^{-4} \\
&<0.5 \times 10^{-4} \\
&\end{aligned}$ & $\begin{array}{r}116.2 \\
110.3 \\
110.3 \\
73.4 \\
251.1 \\
144.2 \\
249.3 \\
\end{array}$ & $\begin{array}{r}189.0 \\
185.1 \\
185.1 \\
71.6 \\
224.6 \\
202.4 \\
223.6 \\
\end{array}$ & $\begin{array}{l}-0.79 \pm .09 \\
-0.05 \pm 0.08\end{array}$ & & & \\
\hline$\overline{\Sigma^{0}}$ & $\Delta y$ & $100 \%$ & 77.0 & 74.5 & & & & \\
\hline$\Sigma^{-}$ & $\begin{array}{l}n \pi^{-} \\
n \pi^{-} \gamma \\
n \mu^{-} v \\
n e^{-\nu} \\
\Lambda e^{-\nu}\end{array}$ & $\begin{array}{l}100 \% \\
\approx 0.1 \times 10^{-4} \\
(0.66 \pm .15) 10^{-3} \\
(1.2 \pm 0.2) 10^{-3} \\
(0.75 \pm .28) 10^{-4} \\
\end{array}$ & $\begin{array}{r}118.1 \\
118.1 \\
152.0 \\
257.1 \\
81.2 \\
\end{array}$ & $\begin{array}{r}192.8 \\
192.8 \\
209.4 \\
229.9 \\
79.0 \\
\end{array}$ & $-0.16 \pm .21$ & & & \\
\hline$\Xi^{0}$ & $\begin{array}{l}\Lambda \pi^{0} \\
p \pi^{-} \\
p e^{-\nu} v \\
\Sigma^{+} e^{-v} v \\
\Sigma^{-} e^{+} v\end{array}$ & $\begin{array}{l}\approx 100 \% \\
<2.7 \% \\
<2.7 \% \\
<1.3 \%\end{array}$ & $\begin{array}{r}63.9 \\
236.5 \\
375.5 \\
124.4 \\
116.6 \\
\end{array}$ & $\begin{array}{l}134.8 \\
298.7 \\
322.2 \\
119.0 \\
111.9 \\
\end{array}$ & $-0.34 \pm .12$ & 0.05 & 0.94 & $(8 \pm 62)^{\circ}$ \\
\hline$\overline{\Xi^{-}}$ & $\begin{array}{l}\Lambda \pi^{-} \\
\Lambda \mathrm{e}^{-\nu} \\
\mathrm{n} \pi^{-}\end{array}$ & $\begin{array}{l}100 \% \\
\leqslant 1.7 \times 10^{-3} \\
<5 \times 10^{-3} \\
\end{array}$ & $\begin{array}{r}65.8 \\
204.9 \\
241.7 \\
\end{array}$ & $\begin{array}{l}138.7 \\
189.4 \\
303.0 \\
\end{array}$ & $-0.410 \pm .046$ & +0.12 & 0.90 & $(-17 \pm 18)^{\circ}$ \\
\hline
\end{tabular}

The definition of the se quantities is taken as follows:
$a=\frac{2 \operatorname{Re}\left(S^{*} P\right)}{|S|^{2}+|P|^{2}}$
$\beta=\frac{2 \operatorname{Im}\left(S^{*} P\right)}{|S|^{2}+|P|^{2}}$
$\gamma=\frac{|\mathrm{S}|^{2}-|\mathrm{P}|^{2}}{|\mathrm{~S}|^{2}+|\mathrm{P}|^{2}}$
$\tan \Delta=\frac{\beta}{a}$.

A. H. Rosenfeld, A. Barbaro-Galtieri, W. H. Barkas, P. L. Bastien, J. Kirz, and M. Roos, UCRL-8030-Part I, August 1965. 

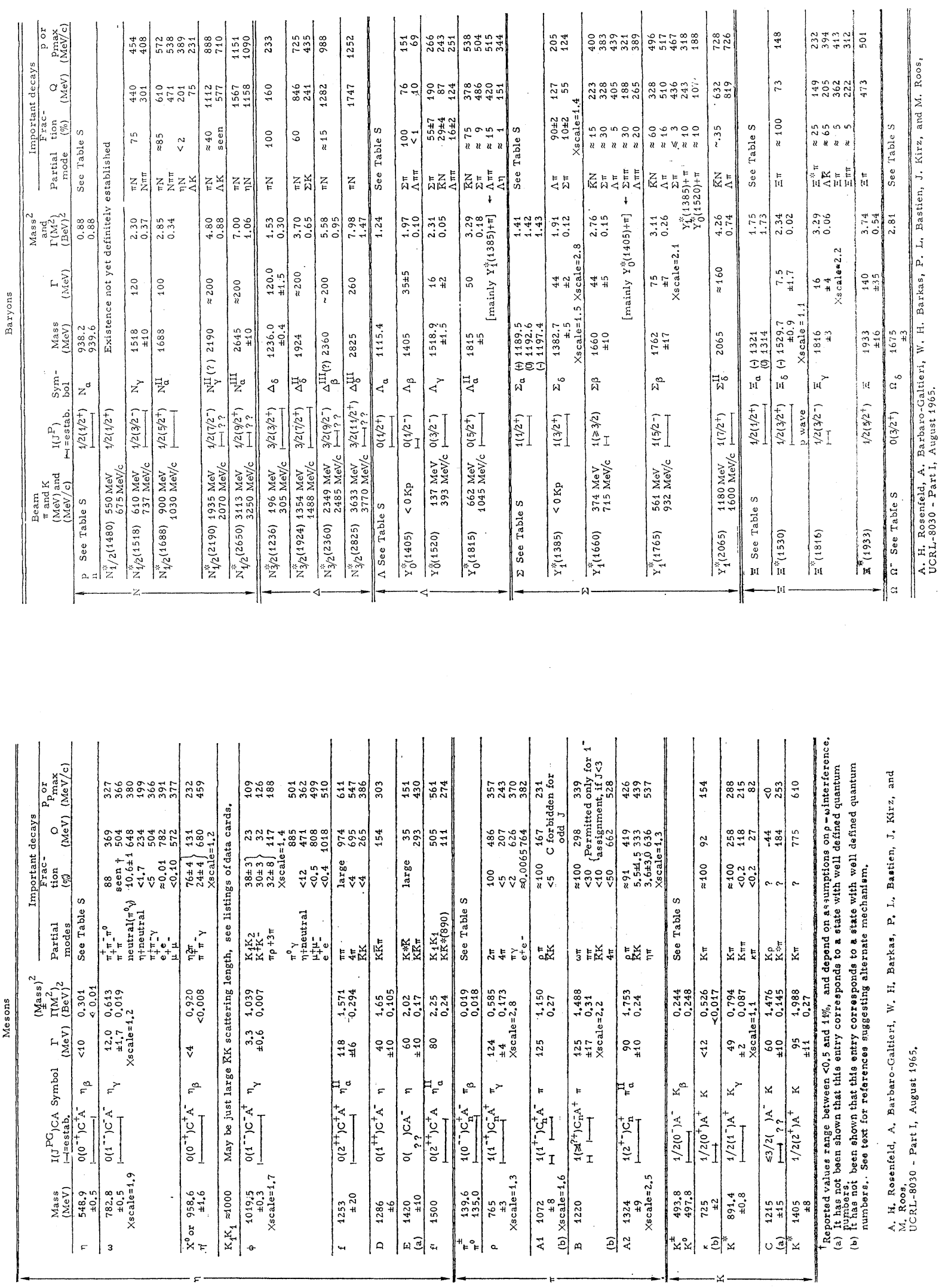
nine $1^{-}$states $\left(\rho, K^{*}(890), \omega, \phi\right)$ are known, and the grouping of $\left(A 2, K^{*}(1440), f^{0}, f^{\prime}\right)$ into a $2^{+}$nonet has been suggested." These nonets may be considered as (octet+singlet) representations of $\mathrm{SU}(3)$, with possible mixing between the isosinglet member of the octet and the $\mathrm{SU}(3)$ singlet to form the observed particles. The Gell-Mann-Okubo formula

$$
M^{2}{ }_{I=0}=\frac{1}{3}\left[4 M_{I=\frac{1}{2}}^{2}-M_{I=1}^{2}\right]
$$

${ }^{11}$ L. M. Hardy et al., Phys. Rev. Letters 14, 401 (1965); R. C. Arnold, Phys. Rev. Letters 14, 657 (1965); S. L. Glashow and R. H. Socolow, Phys. Rev. Letters 15, 329 (1965); also, S. U. Chung"et al., Phys. Rev. Letters 15, 325 (1965); and V. E. Barnes et al., Phys. Rev. Letters 15, 322 (1965). predicts the mass of the $I=0$ member of the octets. Note that in all three cases the calculated mass falls between the masses of the two observed $I=0$ states in the nonet as is required. For $0^{-}$mesons the predicted value $(568 \mathrm{MeV})$ is close to the $\eta$ mass, and does not require significant mixing between the $\eta$ and the $X^{0}$. In the other two cases $\left(928 \mathrm{MeV}\right.$ for $1^{-}, 1435 \mathrm{MeV}$ for $\left.2^{+}\right)$the mixing is considerably stronger.

1. Recent revisions and comments may be found in the review papers by A. H. Rosenfeld and by Ch. Peyrou in the Proceedings of the 1965 Oxford Conference on High Energy Physics (to be published January 1966). 


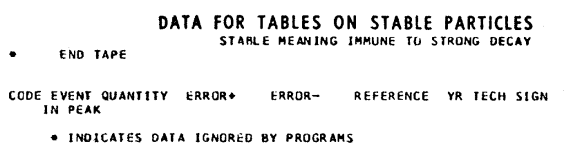

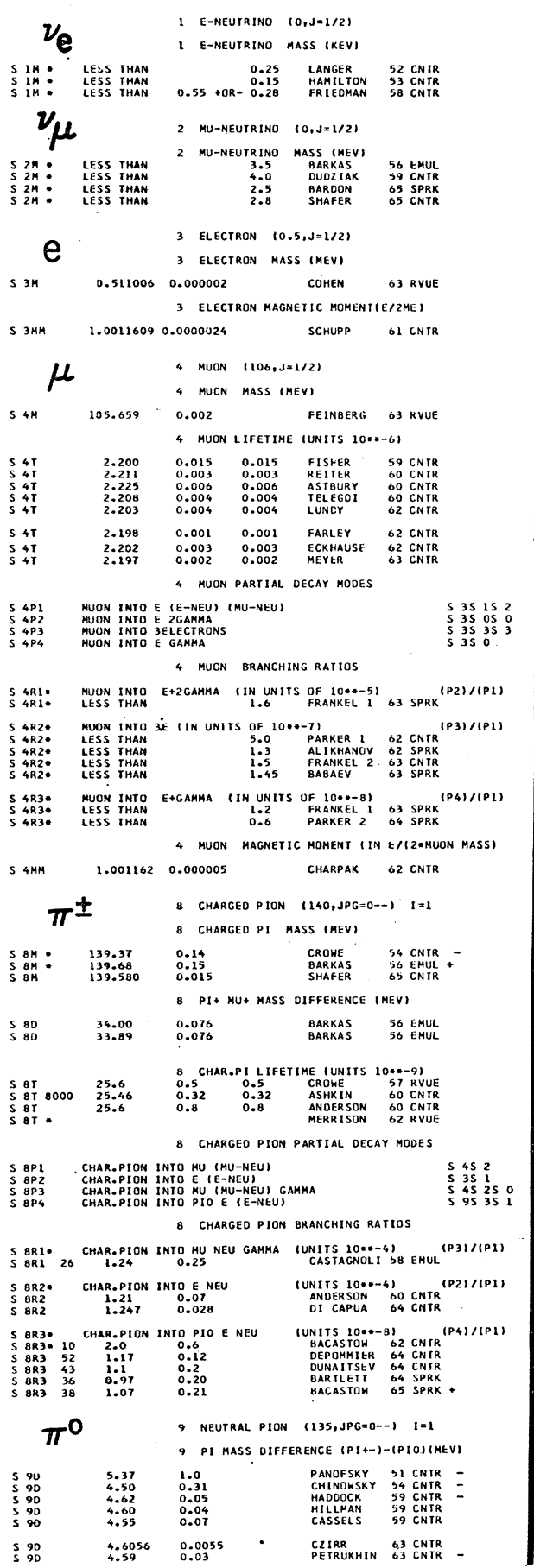

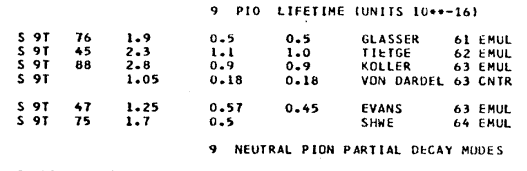

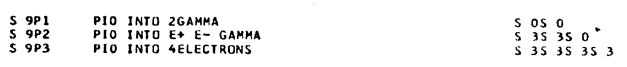

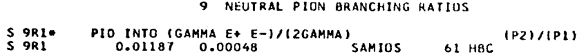

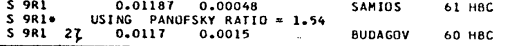

REFERENCES FOR TABLES ON STABLE PARTICLES

10ENTIFIC. Yr AUtHors

$\nu_{e} \quad \cdots$ I E-Neutrino $(0, J=1 / 21$

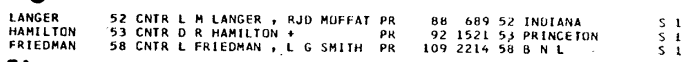

$\boldsymbol{\nu} \boldsymbol{\mu} \quad 2$ mu-neutrino $10, J=1 / 21$

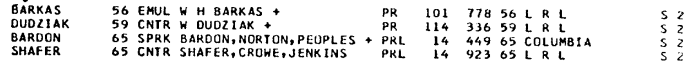

e 3 ELECTRON $10.5, J=1 / 21$

SCHUPP
COHEM

$\mu \quad$ a nuON $(106, j=1 / 21$

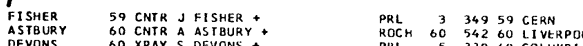

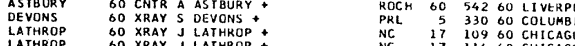

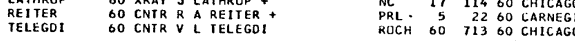

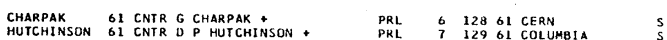

ALIKHANOV 62 SPRK A I ALIKHANOV * CERM 42362 ITEP

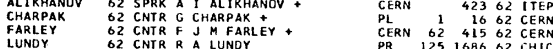

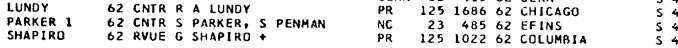

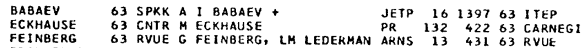

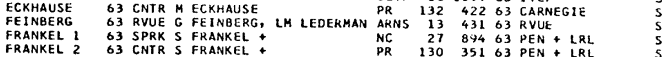

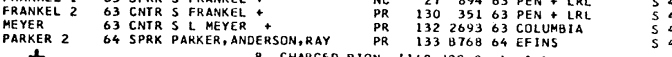

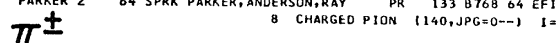

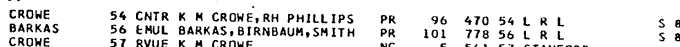

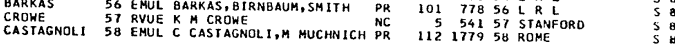

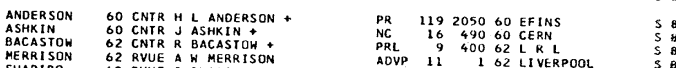

ADVP
PRP

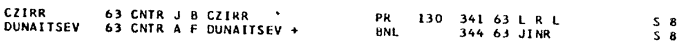

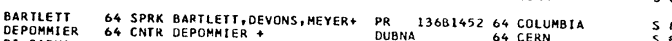

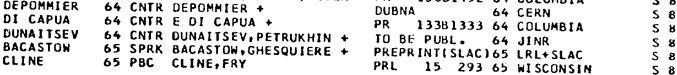

$\pi^{\mathbf{O}} \quad$, NEUTRAL PION 1135, JPG $=0--1 \quad t=1$

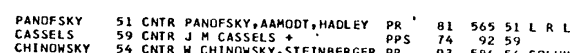

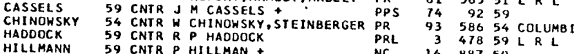

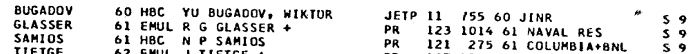

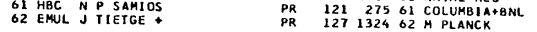

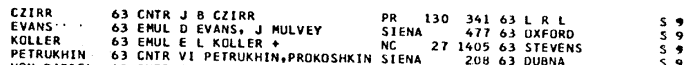




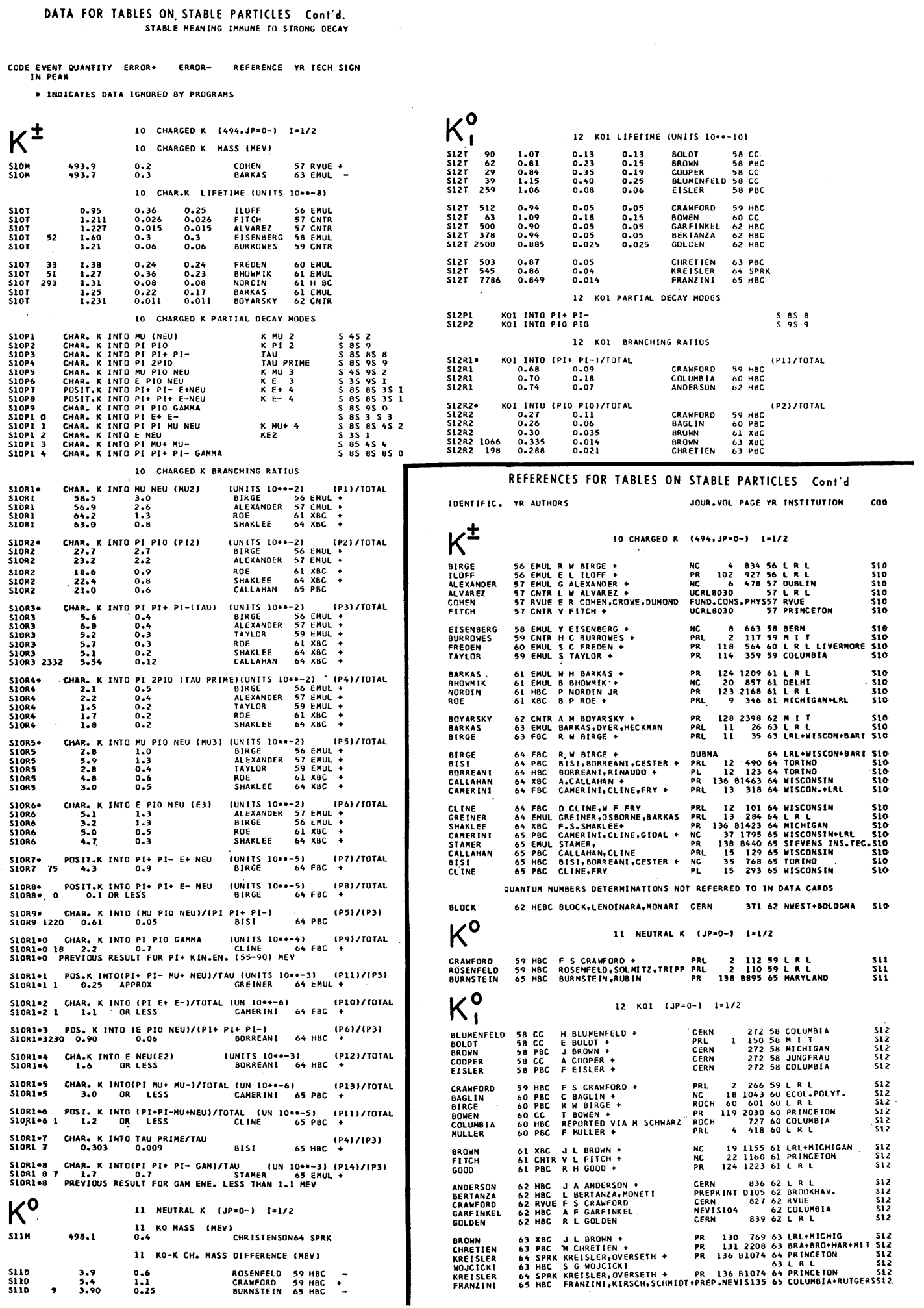


DATA FOR TABLES ON STABLE PARTICLES COnt'd.

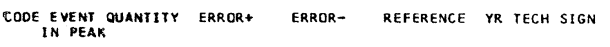

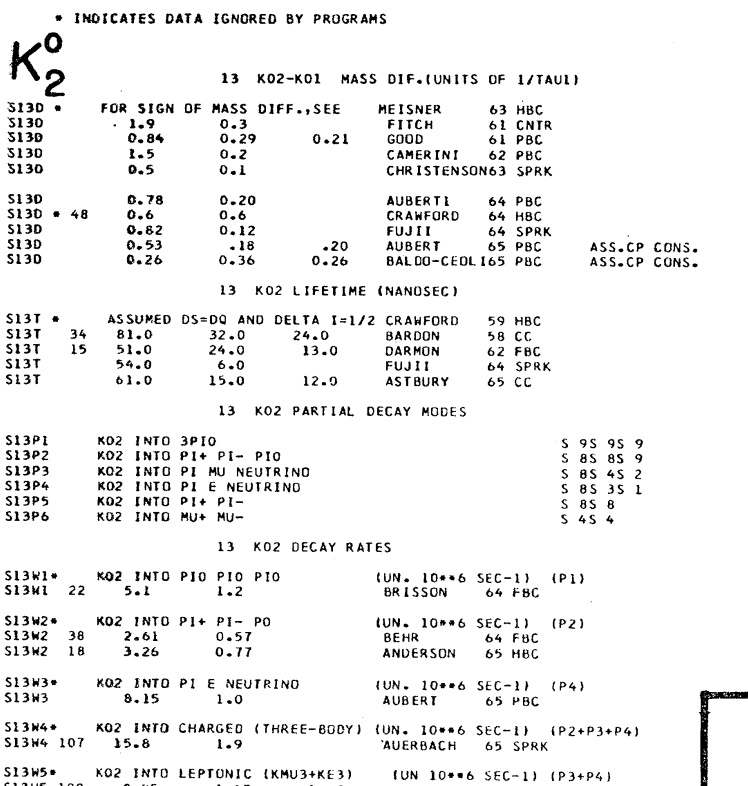

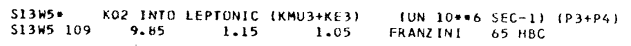
13 KO2 BRANCHING RATIOS

S13R1.
S13R1

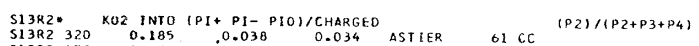

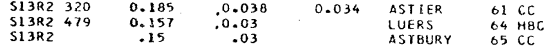

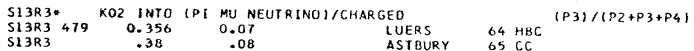

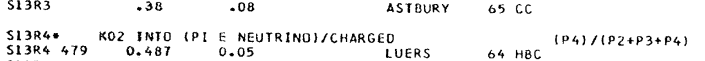

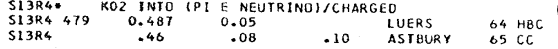

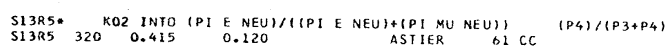

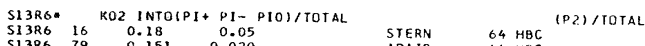

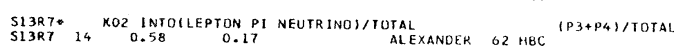

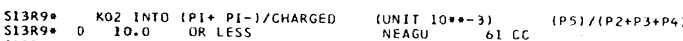

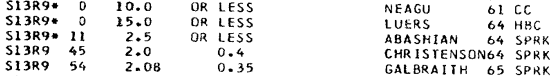

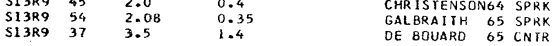

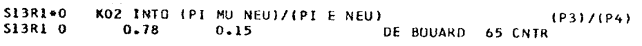
${ }_{S 13 R 1010}^{S 13 R 101} \begin{gathered}\text { KO2 } 2 \text { INTO (MU+MU-)/CHARGED } \\ \text { SR LESS }\end{gathered}$

$\eta$ $\begin{array}{lrr}S 14 M & 53 & 549.0 \\ S 14 M & 35 & 546.0 \\ S 14 M & 91 & 54.0 \\ S 14 M & 549.0 \\ S 14 M & 148 & 549.0 \\ S 14 M & 325 & 552.0\end{array}$ 14 ETA $\{549, J P G=0-+1 \quad I=0$ 14 ETA MASS (MEV)

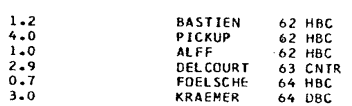

14 ETA WIDTH (MEV)

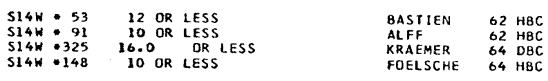

14 eta partial decay modes

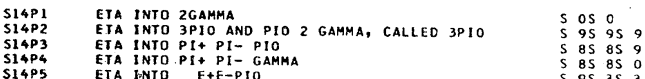

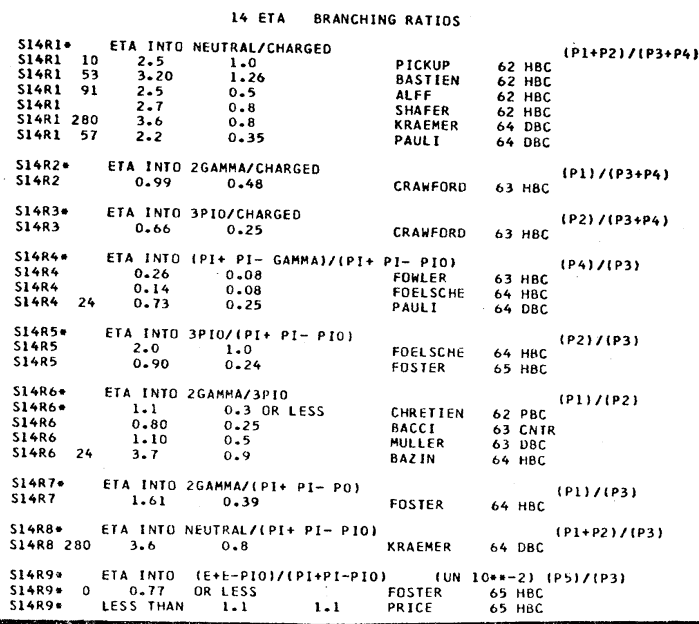

REFERENCES FOR TABLE ON STABLE PARTICLES Cont'd.

IDENTIFIC. YR AUTHORS JOUR.VOL PAGE YR INSTITUTION COD

\section{KO $\quad 13$ KO2 $\quad(J P=0-1 \quad I=1 / 2$}

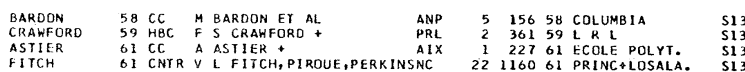

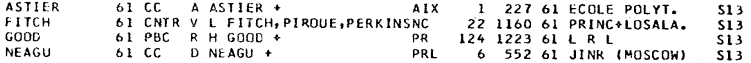

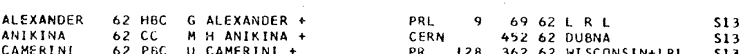

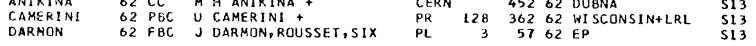

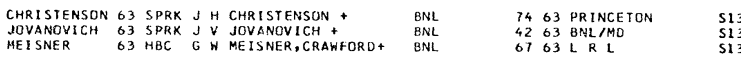

ABASHIAN 64 SPRK ABASHIAN, ABRAMS + PRL 1324364 ILLINOIS S13

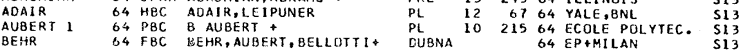

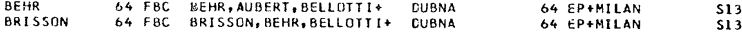

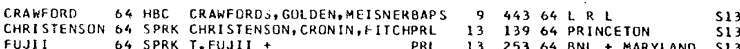

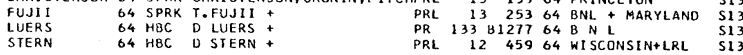

ANDERSON $65 \mathrm{HBC}$ ANDERSON, CRAWFORD, GOLDENPRL $1447565 \mathrm{~L} R \mathrm{~L}, \quad$ L 13

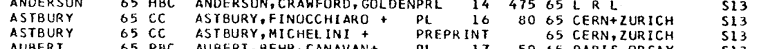

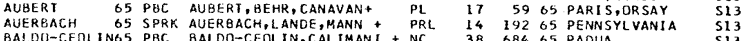

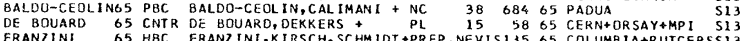

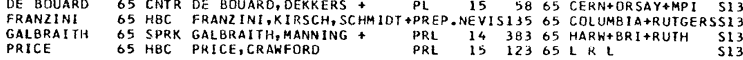

$\eta$

14 ETA $1549, J P G=0-+1 \quad I=0$

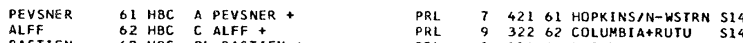

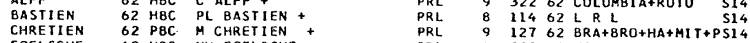

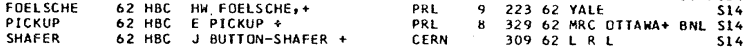

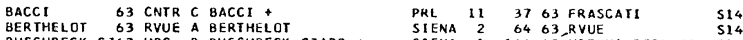

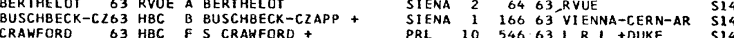

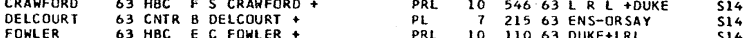

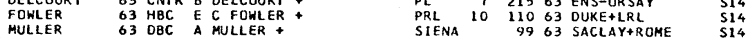

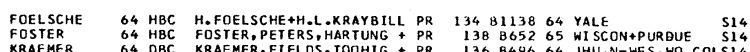

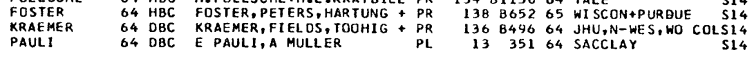
FOSTER O5 HBC FOSTER, GOOD, MEER ATHENS 65 HISCONSIN, PURDU SIG CUANTUM NUMBER DETERMINATIONS NOT REFERKED IO IN DATA CARDS

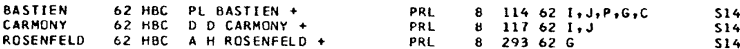


DATA FOR TABLES ON STABLE PARTICLES Cont'd.

CODE EVENT QUANTITY ERROR+ ERrOK- REFERENCE YR TECH SIGN
IN PEAK

- indicates data ignored by programs

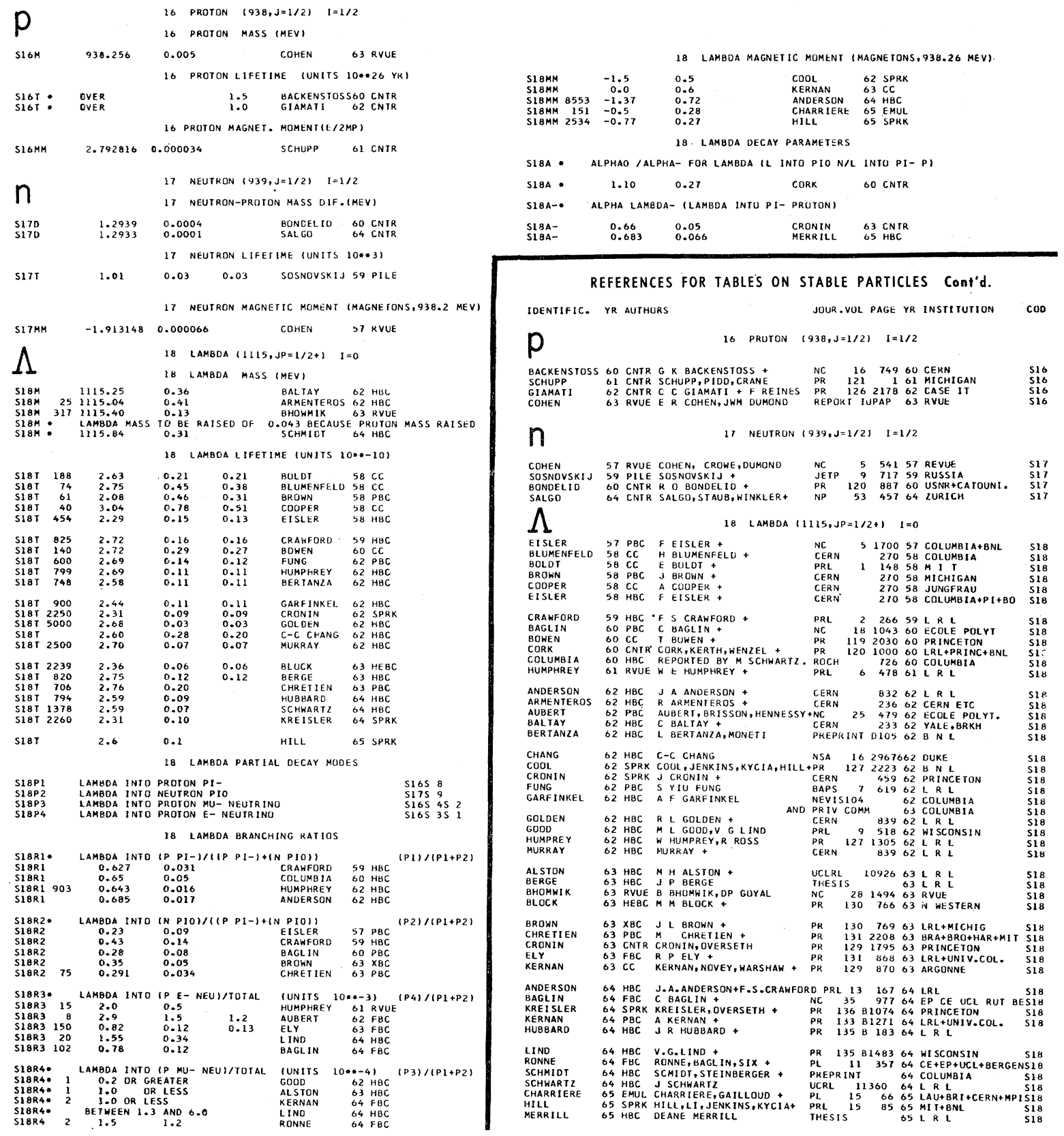


DATA FOR tables ON STABLE PARTICLES COn''d.

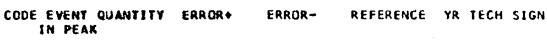

INDICATES DATA IGNOREO QY PROGRAMS

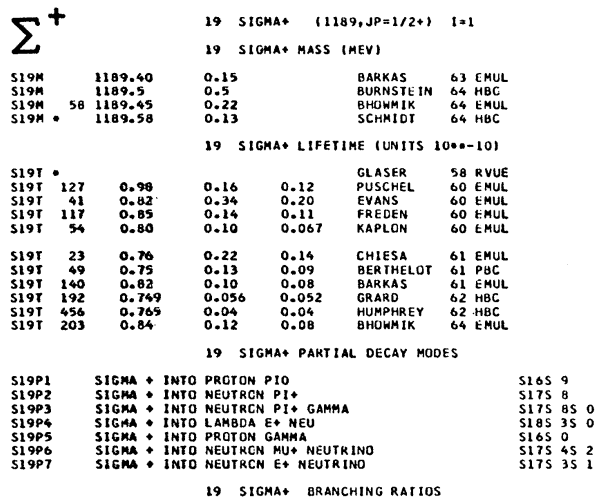

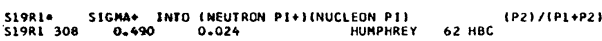

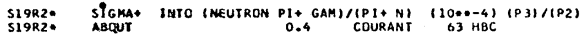

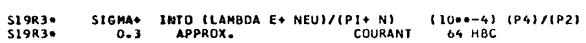

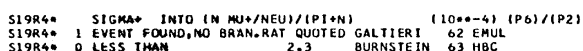

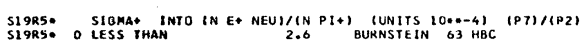

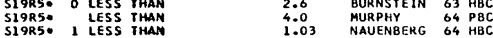

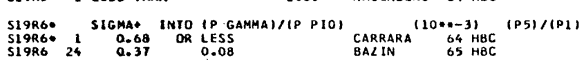

19 SIGMAa MAGNETIC MOMENT (MAGNETONS, 938.26 MEV

$\begin{array}{llll}\text { S19MM } 24 & 4.3 & \text { 1i5 MCINIURFF O4 EMUL } \\ & & 19 \text { SIGHA+ DECAY PARAMETERS }\end{array}$

S19A. ALPHa SIGMat ISIGMA + INTO PI+ NEUTRON)

$\begin{array}{lllll}\text { S19A+: } & -0.03 & 0.08 & \text { CORK } & 60 \mathrm{CNIR} \\ \text { S19A.: } & -0.20 & 0.24 & \text { TRIPP } & 62 \mathrm{HBC}\end{array}$

S19AO. ALPHA SIGMAO ISIG+ INTO PIO PROTOM)

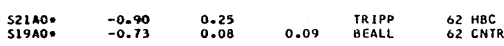

$\sum$ 20 SIGMA- $11198, J P=1 / 2+1 \quad 1=1$

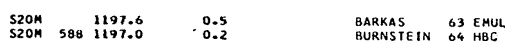

20 SIGMA- MASS DIFFER $(-1-1+1$ (MEV)

$\begin{array}{lllll}5200 & 2500 & 8.25 & 0.25 & \text { DOSCH }\end{array} 65$ HBC

20 SIGMA- LIFETIME IUNITS $10 \cdots-101$

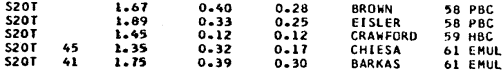

S20T 1208 1.58 0.060 .06 HUMPHREY 62 HBC

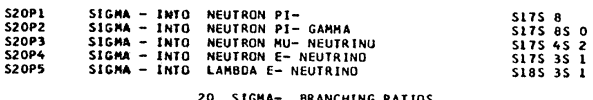

S20R1.
S20R1 22 SIGMA $_{0.06}$ INTO IN MU- NEUI/IN PI-1 CUNIIS $\left.10.0-3\right)$
0.15

S20R2* SIGM - INTO IN E- NEU)/IN PI-1 (UNITS $10.0-3)$ (P4)/(PI)

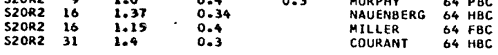

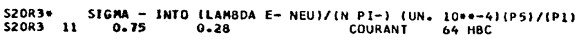

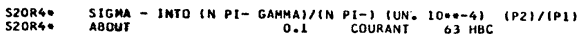

S2OA-: ALPHA S1GMA-
S20A-:
-0.16 20 Sigma- oecar parameters

TRIPP $\quad 62 \mathrm{HBC}$

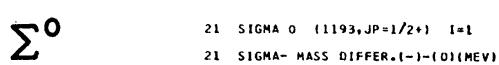

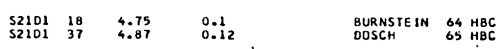

21 SIGMaO LIFETIME IUNITS $10.0-141$
S21T
1.0 OR LESS

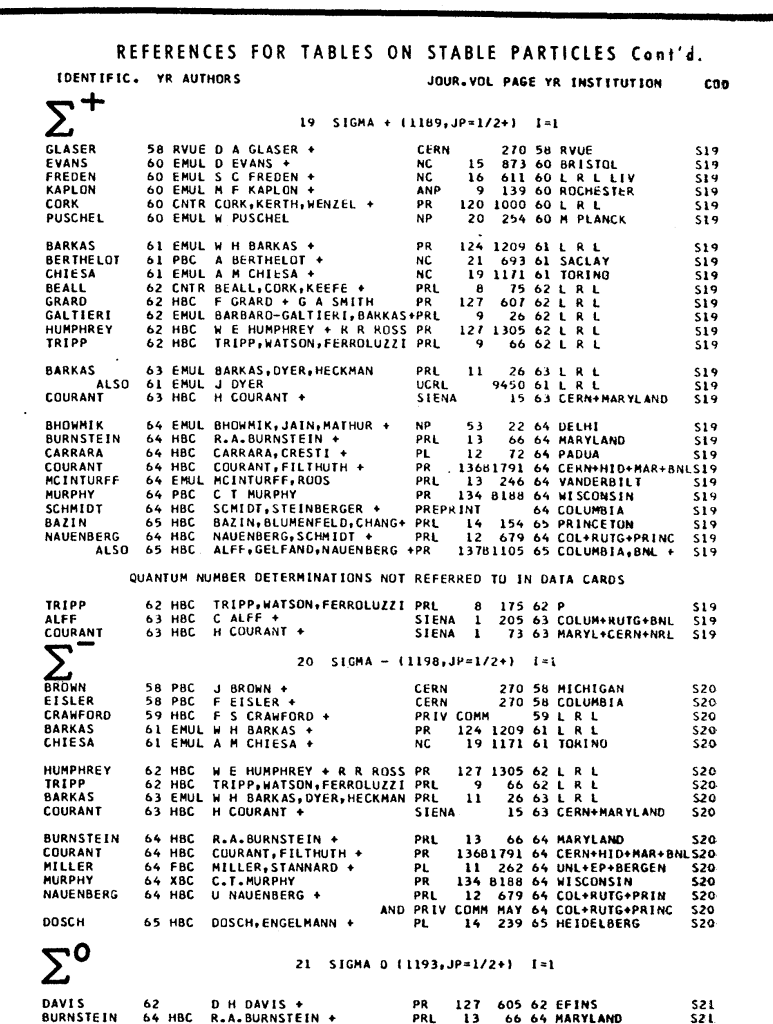

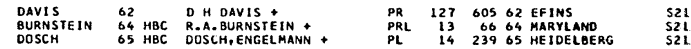




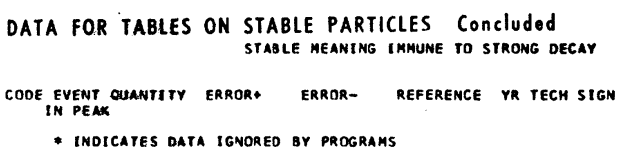

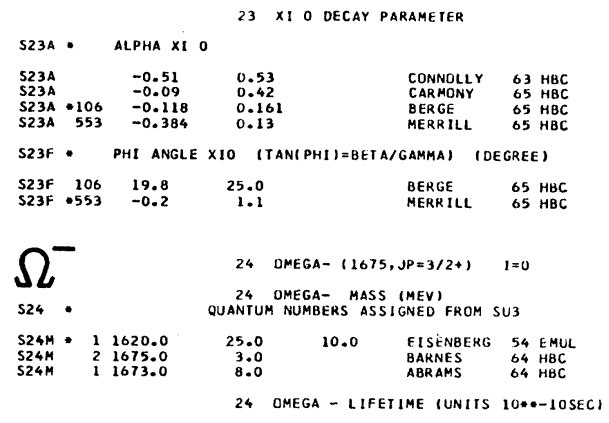




\title{
DATA ON MESON RESONANCES
}

\author{
CODE EVENT OUANTITY ERRUR + ERROR- REFERENCE YR TECH SIGN
IN PEAK
}

- inoicates data ignoreo by programs

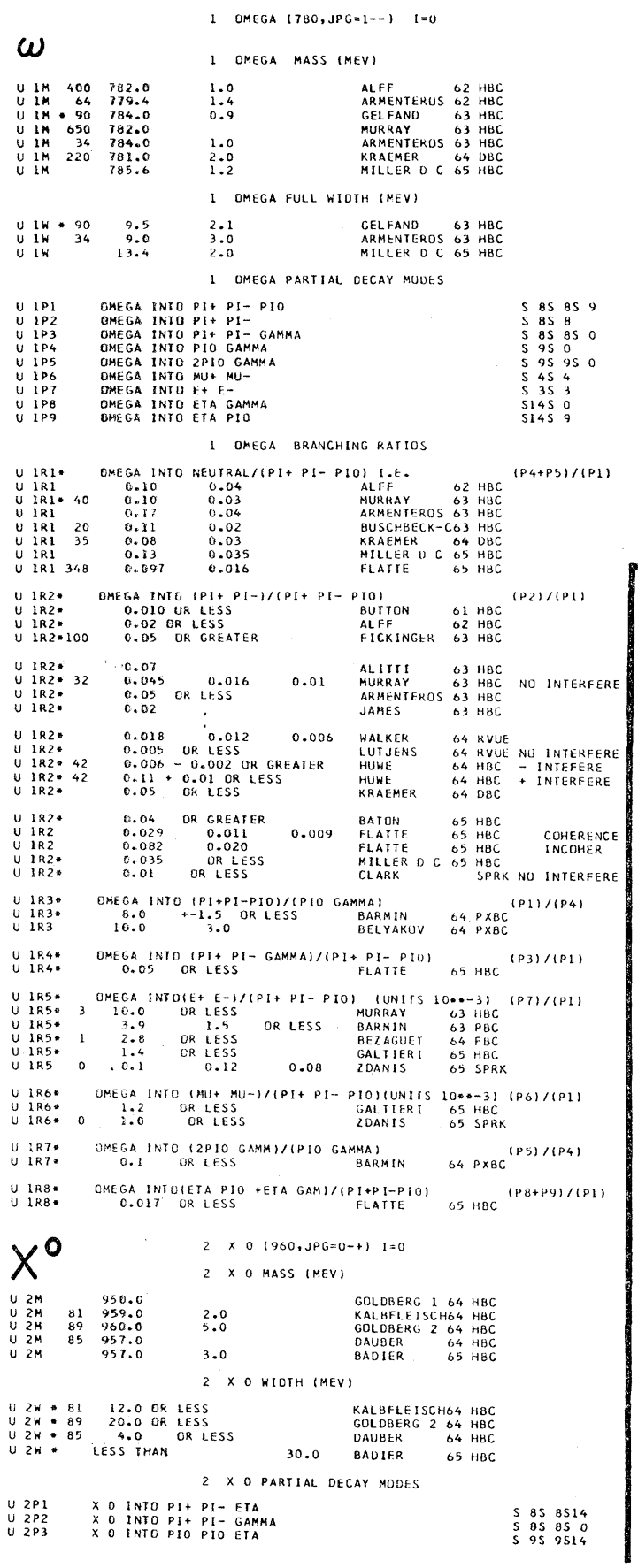

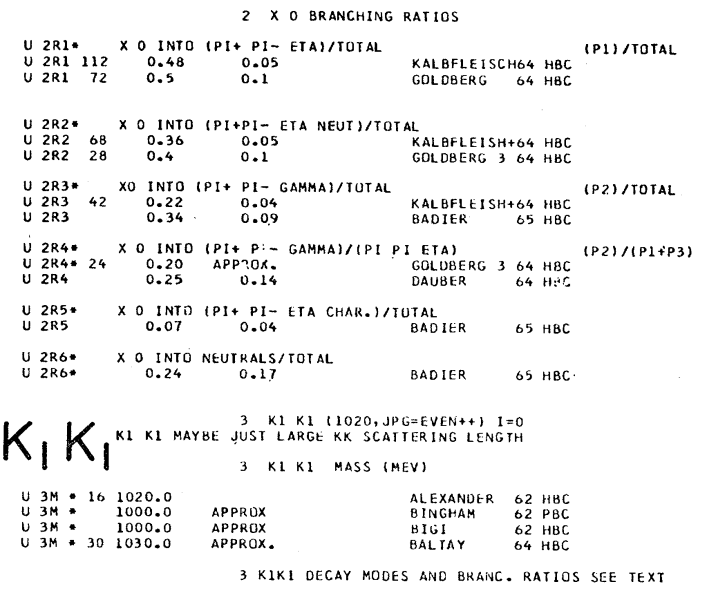

REFERENCES ON MESON RESONANCES

IDENTIFIC. YR AUTHORS

JOUR.VOL PAGE YR INSTITUTION COD

1 OMEGA $(780, J P G=1-) \quad 1=0$

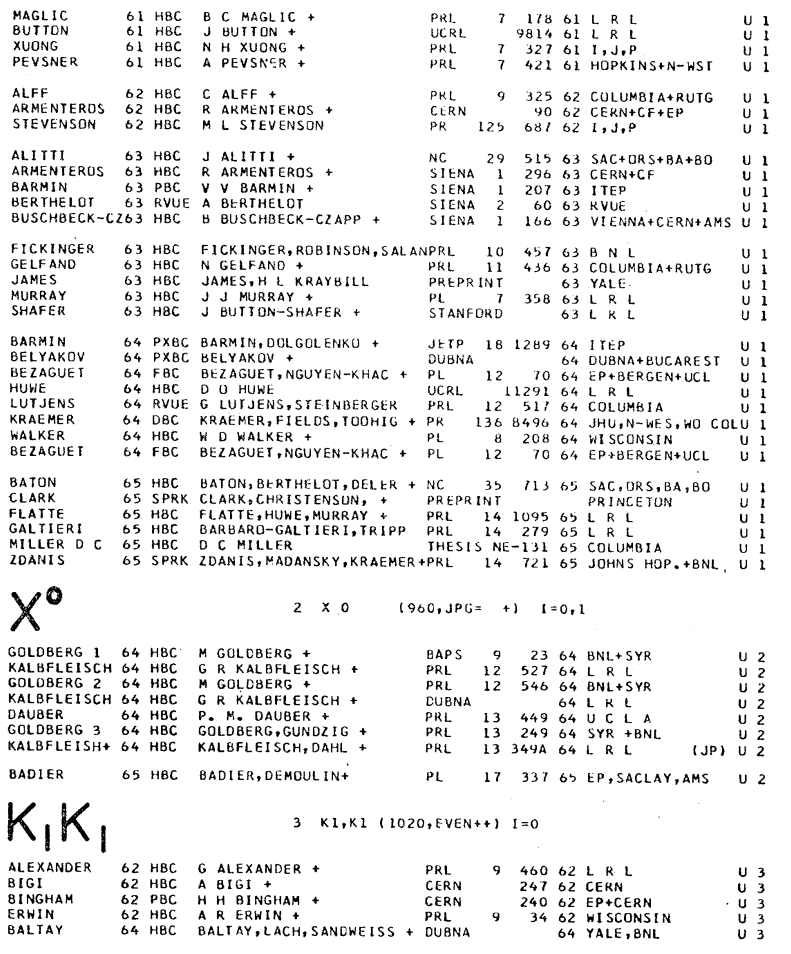




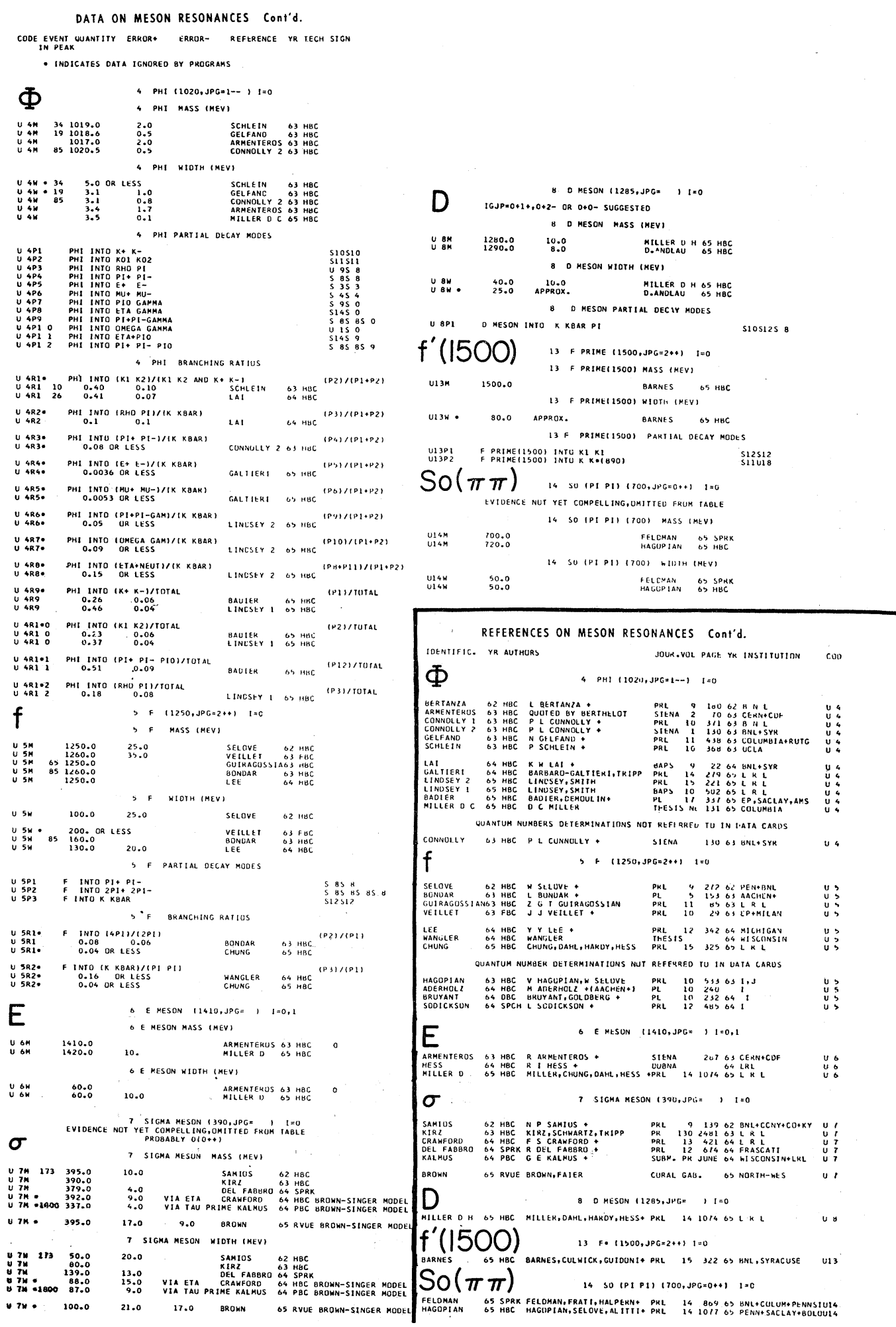




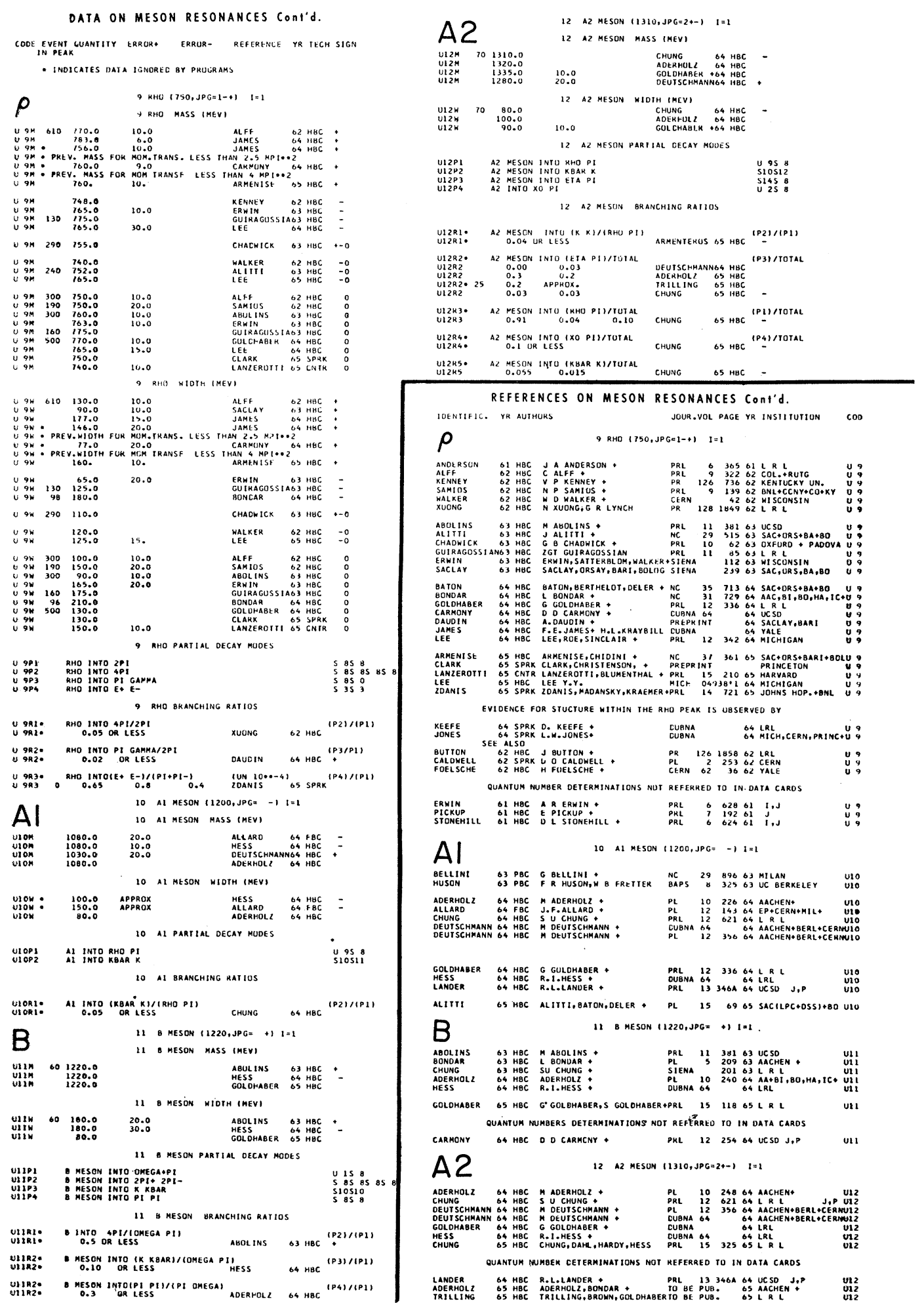




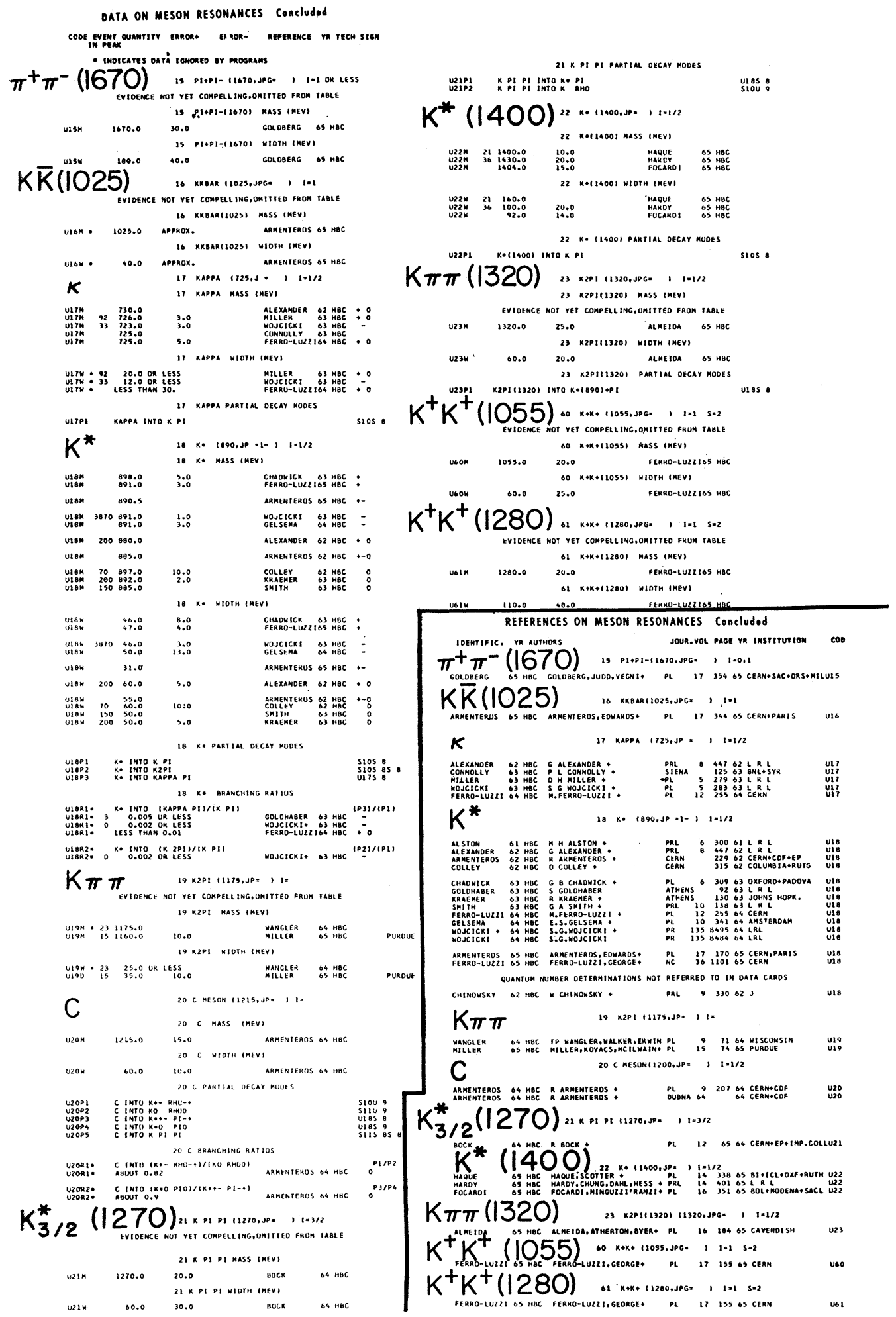


DATA ON BARYON RESONANCES

code event cuantiti errort errur- reference tr technique.

$N_{1 / 2}^{*}(1480)$

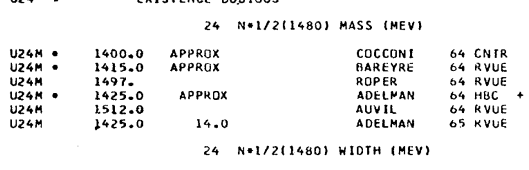

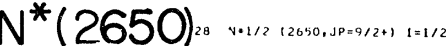

SPIN, PARITY ASSIGNMENT NUT FINAL

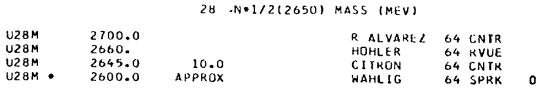

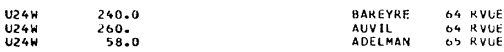

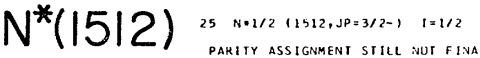
25 N.1/2(1512) MASS (MEV)

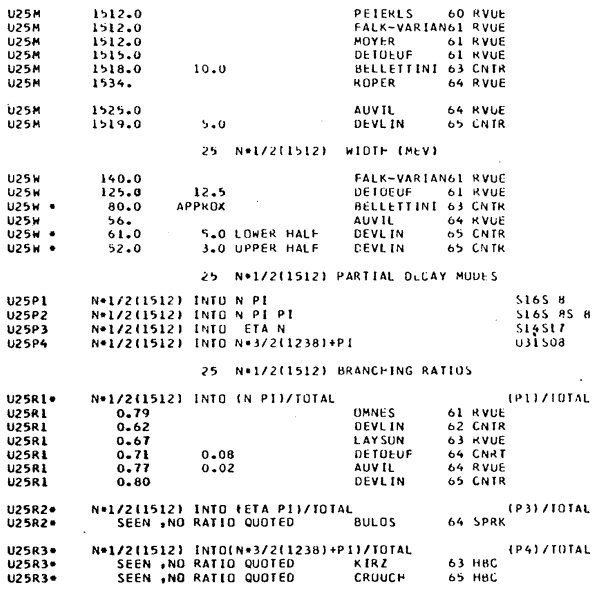

$N^{*}(1688$ 26 N*1/2 $11688, J p=5 / 2+1,1=1 / 2$ PARITY ASSIGNMENT STILL NOT, FINAL 26 N=1/2(1688) MASS (MEV)

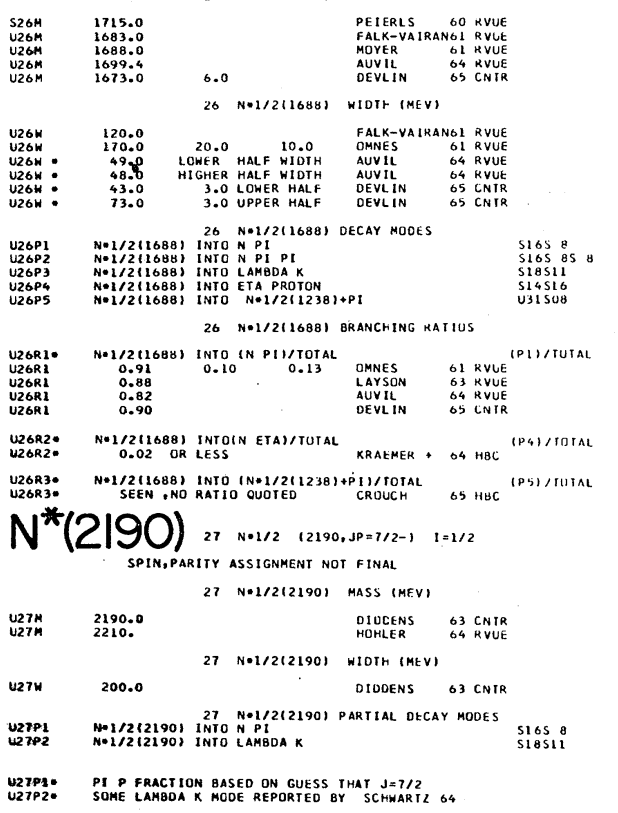

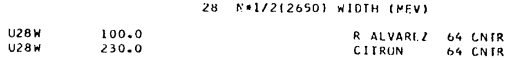

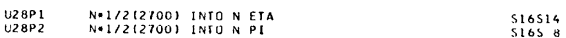

$28 \quad \mathrm{~N}=1 / 212650)$ aranching rastos

U28R1: No1/222700 INTO IN PI)/TOTAL R ALVAR12 G4 CNIR (P2)/TOTAL
U28R1:

REFERENCES ON BARYON RESONANCES

IOENTIFtC. YR aUthIRS JUUR.vUL PAGE YR INSTItUTION COU

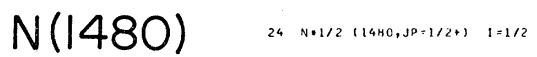

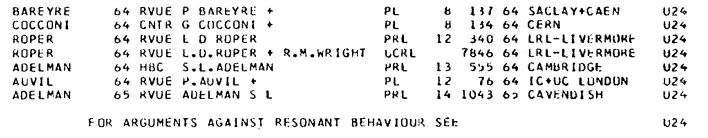

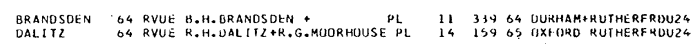

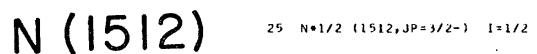

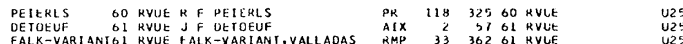

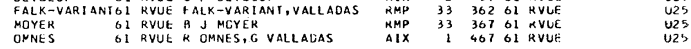

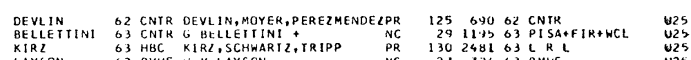

LAYSON O3 RVUE HM IAYSON

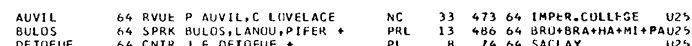

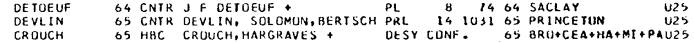

QUantum number determinatiuns not keferkeo to in uata caros

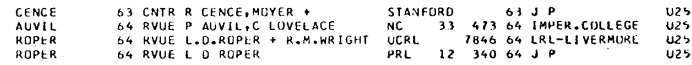

$\mathrm{N}(1688)$

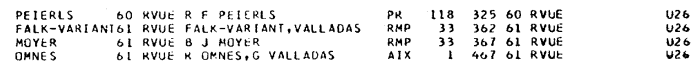

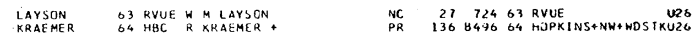

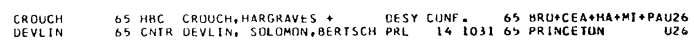
ocantum number oeterminatiuns not kefepred IO IN vata caros

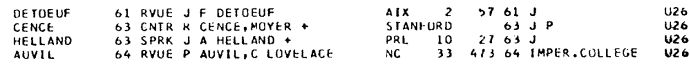

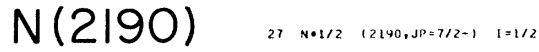

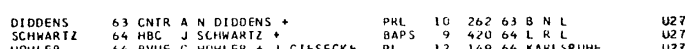

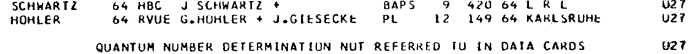

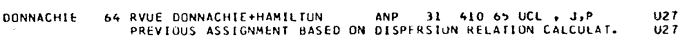

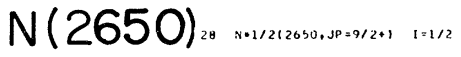

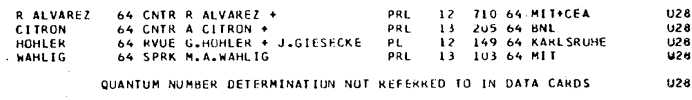

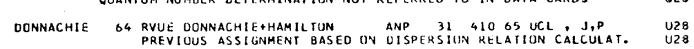



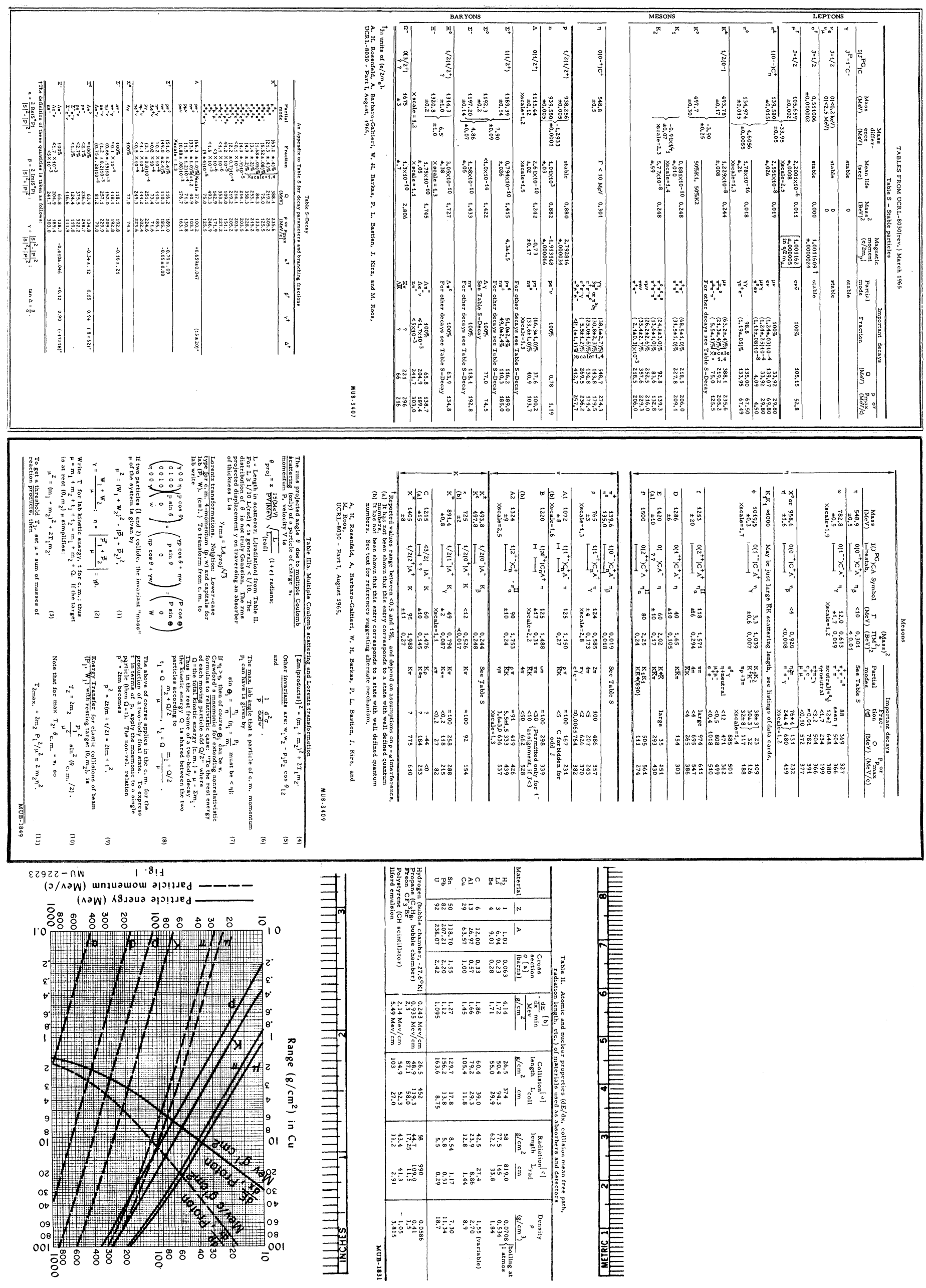

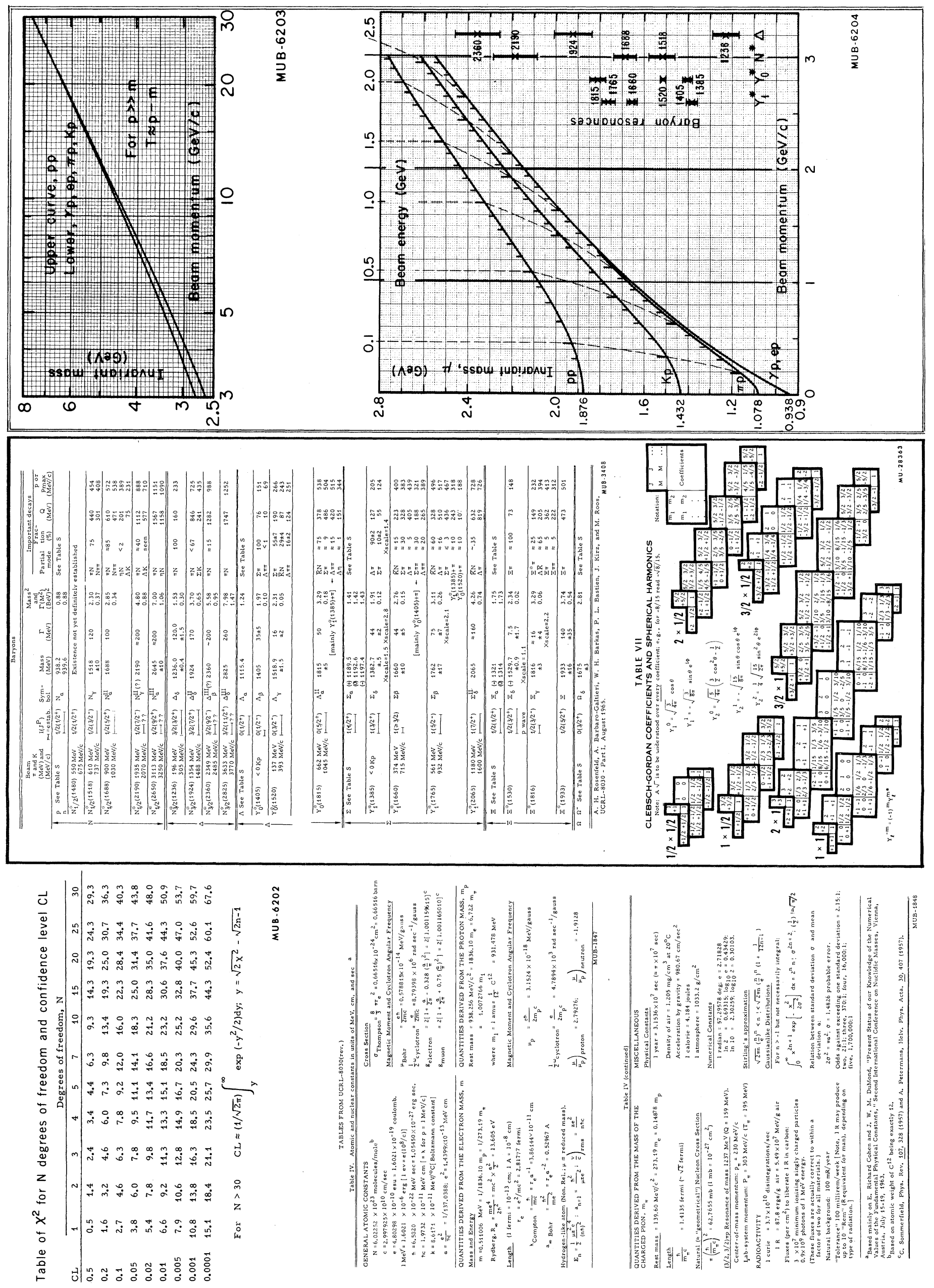
DATA ON BARYON RESONANCES CONI'd.

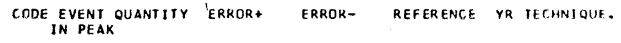

- indicates data ignored gy pkograms

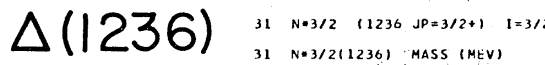

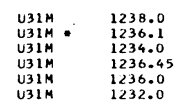

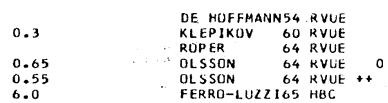

$31 \mathrm{~N} * 3 / 2(1236)$ WIDTH (MEV)

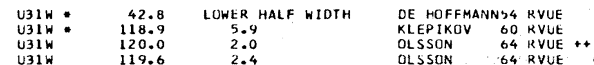

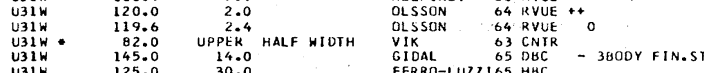

$31 \mathrm{~N} * 3 / 2(1236)$ MASS.DIFF. $(-)-(++)$ (MEV)

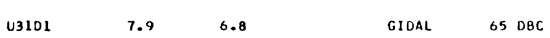

$31 \mathrm{~N} * 3 / 2(1236)$ MASS OLFF. $(0)-(++)$ (MEV)

U3102 0.450 .85 OLSSUN 64 RVUE

U3101*W $25.023 .0 \quad 31 \mathrm{~N} * 3 / 2(1236)$ HALF WIDTH DIFF $(-)-(+1)$ $31 \mathrm{~N} * 3 / 2(1236)$ PARTIAL DECAY MODES

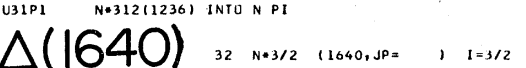
eVIDenCE nOt yet COMPELLING, OMitTEO frum table

$$
32 N=3 / 2(1640) \text { MASS (MEV) }
$$

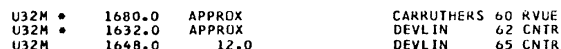
$32 N * 3 / 2(1640)$ WIDTH (MEV)

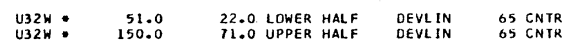

$32 \mathrm{~N}=3 / 2(1640)$ Partial oECAY MUDES

U32P1 $N * 3 / 2(1640)$ INTO $N$ PI
$32 N * 3 / 2(1640)$ BRANCHING RATIOS

U32R1. N*3/2(1640) INTO IN PI)/TOTAL DEVLIN
U32RI
0.56

$\Delta(\mid 920)_{33}{ }_{33}{ }_{N=3 / 2} \quad(1920, J P=7 / 2+1 \quad 1=3 / 2$

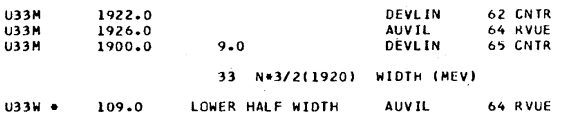

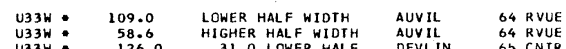

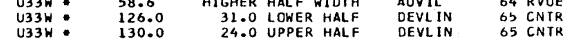

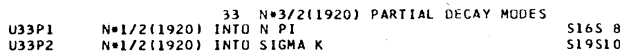

U33P2 N*1/2(1920) INTU SIGMAK $_{33}^{\text {SE3/2(1920) BRANCHING RATIOS }}$

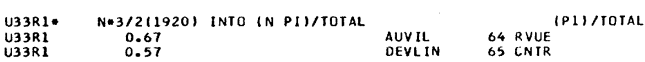

$\Delta(2360)_{34 \quad{ }_{N=3 / 2} \quad(2360, J P=9 / 2-1 \quad 1=3 / 2}$

SPIN, PARITY ASSIGNMENT NOT FINAL

$34 \quad N * 3 / 2(2360)$ MASS (MEV)

$\begin{array}{llllll}\text { U34M } & 2360.0 & & \text { DIDCENS } & 63 \text { CNIR } & \\ \text { U34M } & 2440 . & & \\ \text { U34M. } & 2400.0 & \text { APPROX } & \text { HOHLR } & 64 \text { RVUE } & \\ & & \text { WAHL1G } & 64 \text { SPRK } & 0\end{array}$

$34 N=3 / 2(2360)$ WIDTH (MEV)

U34 200.0 DIDDENS 63 CNTR

$34 N=3 / 2(2360)$ PARTIAL DEGAY MODES

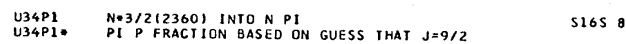

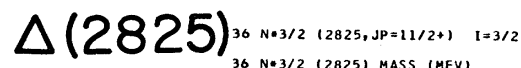

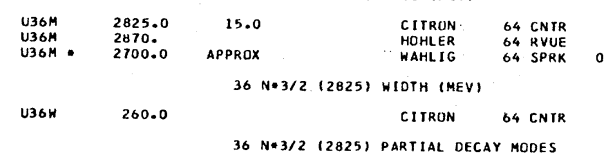

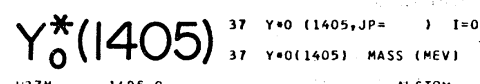

$\begin{array}{llll}\text { U37M } & 1405.0 & \text { ALSTON } & 62 \mathrm{HBC} \\ \text { U37M } & 1405.0 & \text { ALE } & \end{array}$

$37 Y * 0(1405)$ WIOTH (MEV)

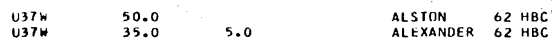

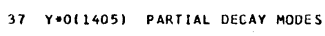

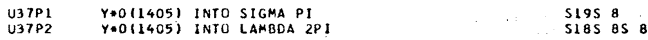

$37 \quad Y * 0(1405)$ BRANCHING RATIUS

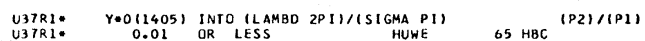

\begin{abstract}
S16S B
\end{abstract}

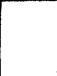

REFERENCES ON BARYON RESONANCES CONT'd.

IOENIIFIC. YR aUthoRs JOUR.VOL PAGE YR INSTITUTION COO

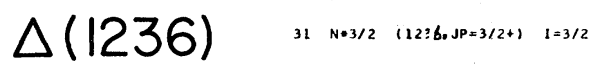

$\begin{array}{llllll}\text { DE HOFFMANN } 54 & \text { RVUE F DE HOFFMANN + } & \text { PR } 951587 & 54 & \text { RVUE } \\ \text { KLEPIKOV OO RVUE N P KLEPIKOV + } & \text { REPORT } & 058460 \text { DUBNA }\end{array}$

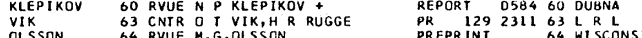

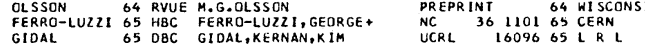

$\triangle(1640) \quad 32 \quad N=3 / 2 \quad 11640, J P=\quad 1 \quad 1=3 / 2$

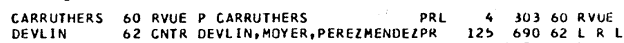

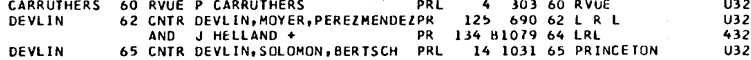

$\triangle(1920) \quad{ }_{33} \quad N * 3 / 2 \quad(1920, J P=7 / 2+1 \quad t=3 / 2$

DEVLIN 62 CNNR DEVLIN, MOYER, PEREZMENDELPR 12509062 PR 1348107964 LRL

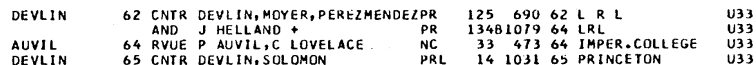

$\triangle(2360) \quad{ }_{34} \quad N * 3 / 2 \quad 12360, J P=9 / 2-1 \quad I=3 / 2$

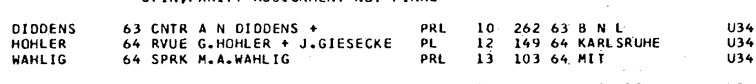

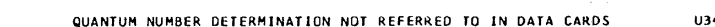

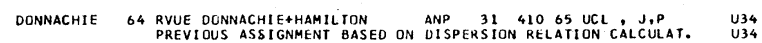

$\Delta(2825)$

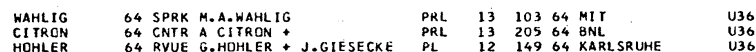
QUANTUM NUMBER DETERMINATION NUT REFERRED TO IN DATA CARDS

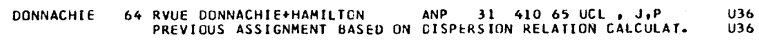

$Y_{0}^{*}(1405) \quad 37 \quad r=0 \quad 11405, J p=\quad, \quad 1=0$

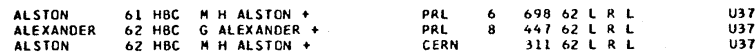
ouantum number determinations nut referred to in data cards

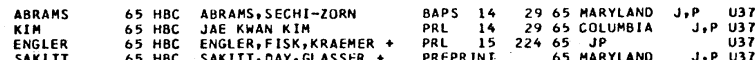




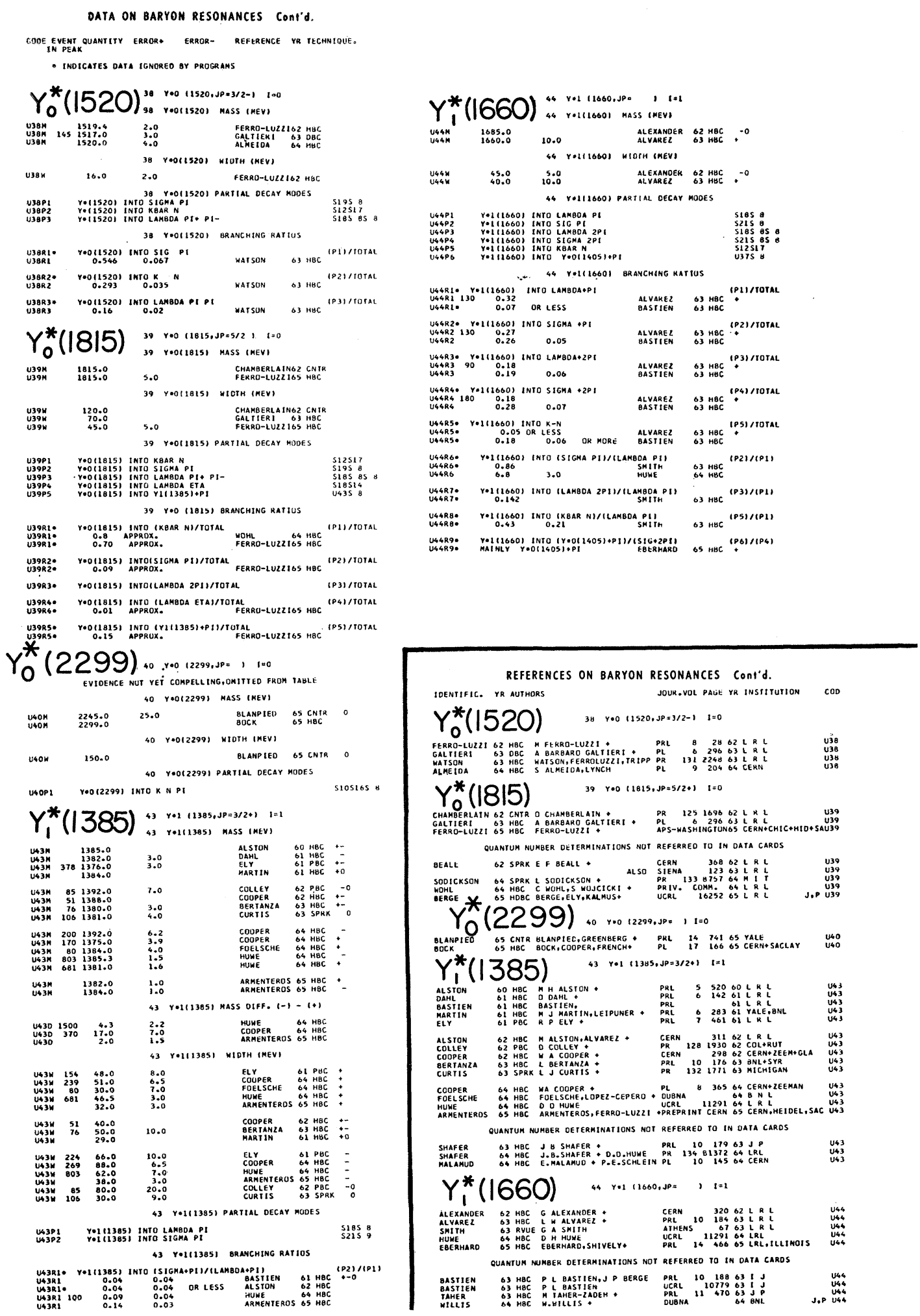


DATA ON BARYON RESONANCES Concluded

COOE EVENT QUANTITY ERror, ERror- reference vr technt QUe.

- inotcates oata ignoreo or prograns

$Y_{i}^{*}(1765)_{\text {45 }}^{45}$ reli1i76s, mass imevi

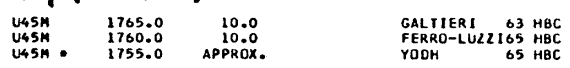
45 Yo111765) WHOTH (MEV)

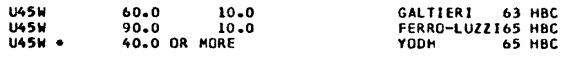
45 Y 1117051 partial decay modes

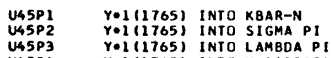

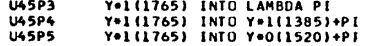

45 Yo1(1765) BRANCHING RATIOS

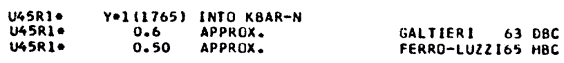

USSR2: Y91 (1765) INTO (SIGMA PI)/TOTAL
US5R20
0.03 OR LESS

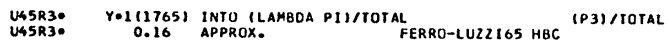

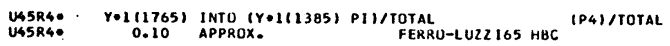

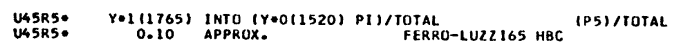

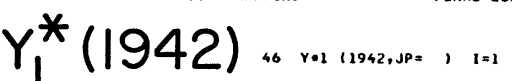

EVIDENCE NOT Yet COMPELLING, OMITIED FROM TABLE

$$
46 \text { Yo1(1942) MASS (MEV) }
$$

U46M 1942.0 BOCK $65 \mathrm{HBC}$

U46P1 Yo1(1942) INrO XN PI

\section{51958
51958
1435
0398
438}

(PI)/TOTAL

(P2)/TOTAL

$$
\text { . }
$$

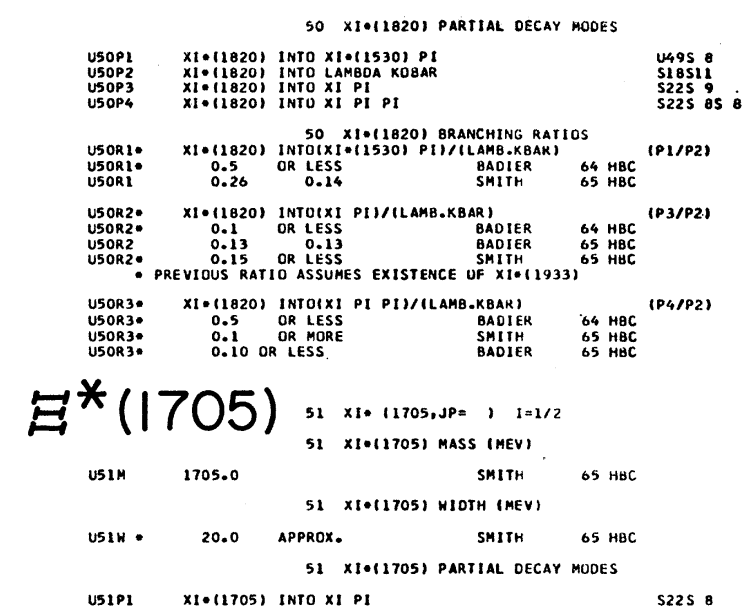

U51P1
USIP2
X1::(11705) INTO X1 P1
XNTO LAMBOA XBAR

52258
$S 18 S$

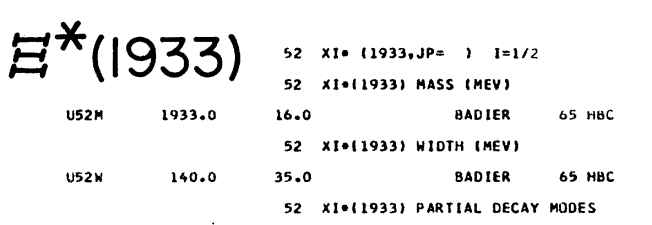

U52P1 X1. (1933) INTO XI PI S22S 8

IDENIIFIC. YR aUthors JOUR.vOL PAGE YR IMSTITIITION COO

$Y_{1}^{*}(1765) \quad$ 45 re1 $11765, \mathrm{sp=5/2}, \quad i=1$

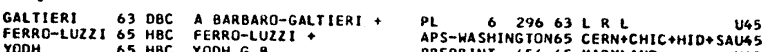

ouantum number determinations not referred to in data caros uas

OERGE 65 HOBC BERGE, ELY, KALMUS + UCRL. 16252 GS LRL JP U4S

$Y_{1}^{*}(1942)$ 46 Y.1 11942,sp= , i=1

BOCK 65 HaC BOCK, COOPER, FRENCH* PL 1716665 CERN SACLAY U46

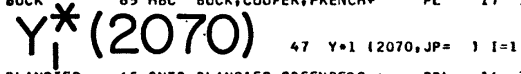

BLANPTEO 65 CNTR BLANP1EO, GREENBERG. PRL 1474165 YALE ULT

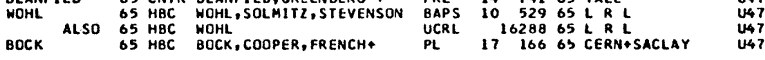

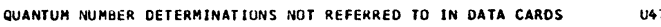

STEVENSON OS HAC STEVENSON. BOULDER CONF. O5 LRL JP U47

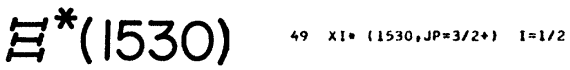

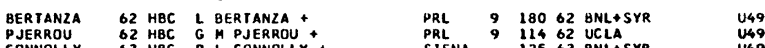

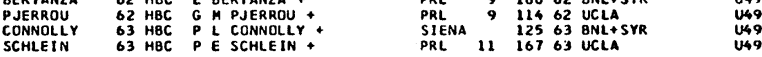

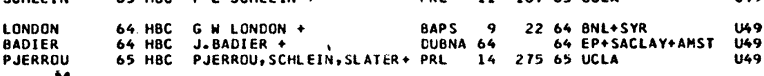

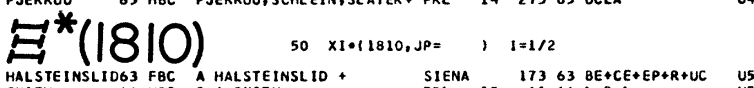

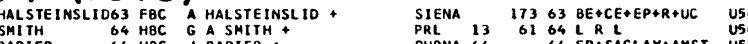

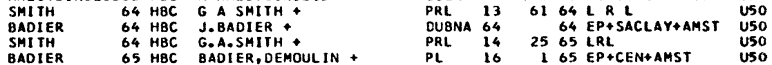

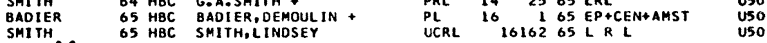

$\Leftrightarrow *(1705)^{51} \times 1 \cdot(1705, \mathrm{JP}=1,1=1 / 2$

SMITH GS HaC SMITH,LINOSEY UCRL 1616265 L RL L USI

$\underbrace{*} *(1933)_{52} \times 1 \cdot 11933, \mathrm{sp}=1, \mathrm{t}=1 / 2$

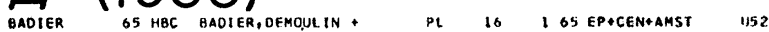

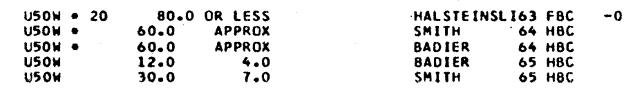

